\title{
Variability of Heavy Metals in Wild Catfish Clarias gariepinus Collected South and North Cairo Area. \\ II. Essential Heavy Metals.
}

\section{Esam H. Rizkalla ${ }^{\circledR}$, Hany H. Haleem and Venees F. Yassa.}

Biochemistry, Nutritional Deficiency Diseases and Toxicology Dept., Animal Health Res. Inst., Agri. Res. Cen., Dokki, Giza, Egypt. e-mail: esamrizkalla@hotmail.com

\section{ABSTRACT}

Four surveys were undertaken to investigate the bioaccumulation of $\mathrm{Fe}, \mathrm{Cu}$, $\mathrm{Zn}$ and $\mathrm{Mn}$ in the liver, kidney, gills, skin and muscles of Clarias gariepinus from the River Nile south (Al-Aiat) and north (Barrage) areas of Cairo. Metals bioaccumulation were analyzed then applied to differentiate between the concentrations found at the two locations, between all of the surveys, and the two sexes.

No significant seasonal variations detected in the water metals. $\mathrm{Fe}$ and $\mathrm{Cu}$ are the highest elements and $\mathrm{Mn}$ is the least element. $\mathrm{Cu}$ is the more element affected by the locality with higher concentrations in Al-Aiat than Barrage. No hazardous effect on the environmental ecosystem observed.

Higher concentrations of metals were observed in the tissues of C. gariepinus than the water in which they live. All heavy metals in all organs are present in measurable quantities and still within the safe limits for consumption.

Concentrations of the analyzed metals revealed large numbers of significant values concerning location, sex and season differences. Discussion is focused on the significant results.

Key words: Clarias gariepinus, Iron, Copper, Zinc, Manganese, Season, Location, Sex.

\section{INTRODUCTION}

Iron, copper, zinc and manganese are classified as essential heavy metals in human diet because they all play significant roles in metabolic processes (Janssens et al., 2009). These metals are also known to help in the growth and production of aquatic life. Alternatively, these minerals may be harmful to aquatic life, either through acute toxicity or chronic effects from accumulations in the organism over extended periods.

Intense activity in the industrial and agriculture sectors has led to the increased discharges of heavy metals in natural waters (Barlas, 1999 and Jordao et al., 2002). According to Mathis and Kevern (1975), because fish are at the higher levels of the food-web and are usually eaten by man, they are the most sensitive group and used as an indicator of metal bioaccumulation in polluted waters. Many heavy metals are concentrated at different levels in different organs of the body (Rao and Padmaja, 2000) and when metals reach sufficiently high concentrations in body cells they can alter the physiological functioning of the fish (Heath, 1987). For these reasons, it is important to determine the concentrations of heavy metals in commercial fish in order to evaluate the possible risk of fish consumption for human health (Cid et al., 2001).

Copper is one of the most abundant trace metals and for almost all organisms it is an essential micronutrient (Duffus, 1980). It has numerous functions in cellular biochemistry including vital roles in cellular respiration, and as a cofactor for over 30 
different enzymes (Gatlin et al., 1989). Copper is used today as a chemotherapeutic agent in aquaculture (Leatherland and Woo, 1998), but it is also toxic in excess (Handy, 1996). It increased coughing frequency, ventilation rate and oxygen consumption, decreased antibody production, decreased osmolarity and plasma $\mathrm{Na}$ levels, disrupted transepithelial ion exchange, inhibited Na-K-ATPase activity, reduction in viral clearance (Sellers et al., 1975), stomach and intestinal distress, liver and kidney damage, and anemia (USEPA, 1991). It can also destroy both chemoreceptors and mechanoreceptors, and this renders the fish incapable of avoidance behaviour (Gardner and LaRoche, 1973). The harmful toxicity is largely attributed to its cupric $\left(\mathrm{Cu}^{2+}\right)$ forms which is the specie commonly found (Olaifa et al., 2004a). The increase in $\mathrm{Cu}$ pollution can be attributed to geological weathering, atmospheric deposition, municipal and industrial sewage, the discharge of mine tailings and fly ash (the major source of solid $\mathrm{Cu}$ pollution), fertilizer production and algaecide and molluscicide runoff (Moore and Ramamoorthy, 1984).

It is commonly accepted that $\mathrm{Zn}$ is an essential micronutrient required for normal growth and metabolic functions by various fish species (Gatlin et al., 1991). Waalkees (1998) reported that a zinc concentration in fish was significantly correlated with traffic density adjacent to highways. As $\mathrm{Cu}$, domestic sewage, chemical manufacturing, painting and coating, mining operations, extractive metallurgy, nuclear and other industries also release heavy metals to the environment (Jarup, 2003).

Iron is an essential trace element for haemoglobin formation (Friberg et al., 1986), respiratory enzymes (Wetzel, 1983), normal functioning of the central nervous system and the enzymatic pathways of carbohydrate, protein and fat (Adeyeye and Otokiti, 1999). The main source of increased Fe concentrations in the aquatic environment is acid mine drainage, mineral and steel processing and industrial runoff (Förstner and Wittmann, 1983).

Manganese is an important component of many metabolic processes, including the functioning of flavoproteins and the synthesis of mucopolysaccharides, cholesterol and haemoglobin (DWAF, 1996). Beliles (1979) pointed out that the major sources for manganese as air and water pollutant are iron and steel manufacturing and the burning of diesel fuel in the motor cars.

The present study was, therefore undertaken to acquire information about the essential heavy metals ( $\mathrm{Fe}, \mathrm{Cu}, \mathrm{Zn}$ and $\mathrm{Mn}$ ) contents of natural carnivorous catfish (Clarias gariepinus) populations from two different study sites along the River Nile in the Cairo capital area. C. gariepinus was chosen based on the social and economic values in Egypt. Although only muscles of the fishes are eaten, samples of inedible tissues (liver, kidney, gills and skin) were also analyzed in order to establish a baseline for future studies of heavy metal pollution. The results were used to test for the effects of locality of capture, sex of fish, seasonal difference and types of tissue of the fishes on their heavy metals contents.

\section{MATERIALS AND METHODS}

\section{Field collection:}

Two main study sites (Fig. 1) of the River Nile were focused on during this study. The first site, Al-Aiat, is situated on the southern border of the Cairo Capital in close proximity to the industrial town of Helwan. The second site, Barrage, is situated approximately $100 \mathrm{~km}$ northern Al-Aiat in close proximity to the industrial town of Shubra El-Khaymah. Samples of Clarias gariepinus were caught by fishermen using 
trawling nets and collected directly by the researchers from both areas. Fish were captured at the same time of collecting the water samples. Fishing trips to the studied areas were carried out every three months over a period of one year during 2008.

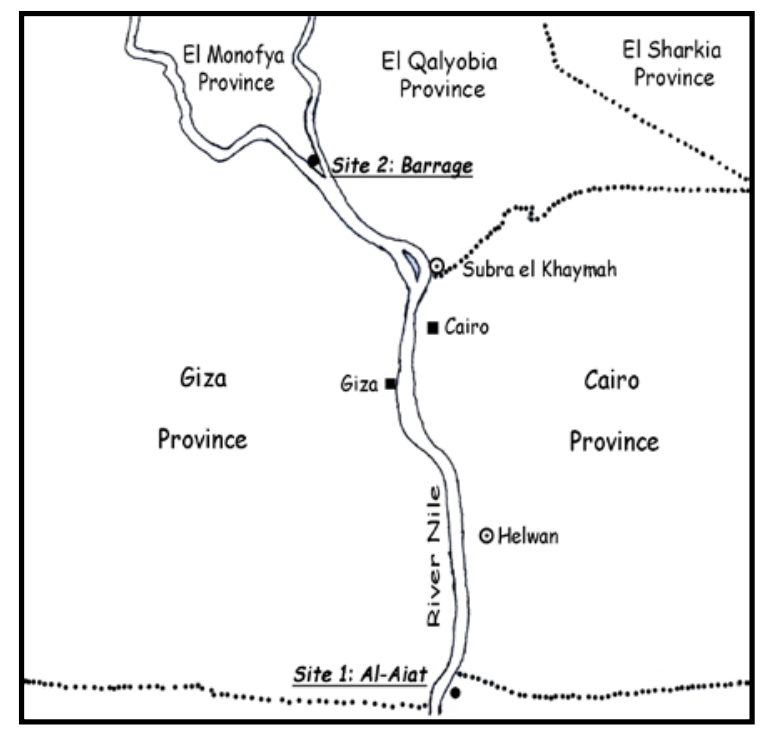

Fig. 1: The location of the two sites studied on the River Nile.

Four water samples from each locality were collected by a Hydro-Bios TPN water sampler at one meter depth as described by Parker (1972) in clean bottles of 1 liter capacity. The water samples were preserved by addition of $1 \mathrm{ml}$ concentrated nitric acid until the time of analysis of heavy metals. The water samples from each site were filtered and kept at $4^{\circ} \mathrm{C}$ until analysis.

All fish were transferred alive in its water into holding tanks to the Animal Health Research Institute. After 24 hours, in the laboratory, measurement of fish standard length was carried out before sampling the organs of fish. The number of fish collected each season and the average total body length $(\mathrm{cm})$ from both fishing sites are outlined in Table 1.

Table 1: Mean \pm Standard deviation of the total body length $(\mathrm{cm})$ of Clarias gariepinus collected from Al-Aiat and Barrage regions.

\begin{tabular}{|c|c|c|c|c|c|c|c|c|}
\hline & \multicolumn{2}{|c|}{$\begin{array}{l}\text { Winter season } \\
\text { January }\end{array}$} & \multicolumn{2}{|c|}{$\begin{array}{l}\text { Spring season } \\
\text { April }\end{array}$} & \multicolumn{2}{|c|}{$\begin{array}{l}\text { Summer season } \\
\text { July }\end{array}$} & \multicolumn{2}{|c|}{$\begin{array}{c}\text { Autumn season } \\
\text { October }\end{array}$} \\
\hline & Male & Female & Male & Female & Male & Female & Male & Female \\
\hline \multicolumn{9}{|l|}{ Al-Aiat region: } \\
\hline No. of samples & 11 & 8 & 8 & 8 & 13 & 7 & 11 & 11 \\
\hline Range & $25-43$ & $25-37$ & $31-41$ & $29-39$ & $22-36$ & $28-33$ & $26-37$ & $22-38$ \\
\hline Mean & 35.6 & 34.5 & 34.7 & 36.3 & 31.9 & 31.1 & 31.4 & 30.9 \\
\hline S.D. \pm & 5.482 & 4.000 & 3.348 & 3.059 & 4.375 & 1.676 & 3.749 & 4.721 \\
\hline \multicolumn{9}{|l|}{ Barrage region: } \\
\hline No. of samples & 10 & 11 & 8 & 8 & 8 & 11 & 12 & 7 \\
\hline Range & $31-40$ & $23-34$ & $34-40$ & $31-42$ & $27-34$ & $25-37$ & $27-40$ & $28-39$ \\
\hline Mean & 35.2 & 30.6 & 36.8 & 35.9 & 31.1 & 30.8 & 32.8 & 31.6 \\
\hline S.D. \pm & 2.974 & 2.911 & 2.188 & 3.300 & 2.295 & 4.094 & 4.366 & 3.780 \\
\hline
\end{tabular}

Each fish was washed with deionized water and dissected to obtain liver, kidney, gills, skin and muscles. The muscle and skin tissue samples were taken from above the lateral line, at the beginning of the dorsal fin in each fish and as deep as the backbone. Gill filaments were removed from the cartilaginous gill arches. After 
dissection, the tissue samples were minced and homogenized separately. The samples were placed on clean dry glass plates and dried in an oven at $105{ }^{\circ} \mathrm{C}$ for 48 hours (Bohn and McElroy, 1976). The dry samples were wrapped in aluminium foil and stored until metal analysis.

\section{Analytical methods:}

Before use, all glassware and plastic utensils were previously soaked overnight in $5 \mathrm{M} \mathrm{HNO}_{3}$ and rinsed with deionized water. One gram of dry samples of the liver, kidney, gills, skin and muscle were digested separately in glass tubes with $10 \mathrm{ml}$ of acid mixture (nitric acid/perchloric acid; 7:3 v/v). The digestion was facilitated by heating at $70^{\circ} \mathrm{C}$. After cooling, all digests were filtered through a Whatman 42 filter paper, and then diluted with deionized water to give a final volume of $25 \mathrm{ml}$.

A Unicam 969 Atomic Absorption Spectrometer with acetylene/air flame was used in accordance with the manufacturer's specifications for the determination of Fe, $\mathrm{Cu}, \mathrm{Zn}$ and $\mathrm{Mn}$ within the digested samples and filtered water (Wood and Van Vleet, 1996).

The percentage of change of metal concentration in a particular organ from Al-Aiat (site I) to Barrage (site II) areas was determined according to the following formula: [(Conc. at site II - Conc. at site I) / Conc. at site I] X 100

\section{Statistical analysis:}

Results were expressed as means \pm standard deviation. Comparisons of means were performed with $t$-tests (Snedecor, 1971). For the smaller subset of data on the bioaccumulation of heavy metals in each tissue, analysis of variance (ANOVA) was used to compare seasonal variability with a fiducially limiting of $5 \%$ (EpiInfo 6.2 and SPSS 8 Softwares). Microsoft excel was used to plot the graphs of the percentage change of heavy metals concentrations in each tissue between the two sites studied.

\section{RESULTS}

\section{Water analysis:}

Range, mean and standard deviation of $\mathrm{Fe}, \mathrm{Cu}, \mathrm{Zn}$ and $\mathrm{Mn}$ concentrations in the water samples collected from Al-Aiat and Barrage areas in the four seasons are summarized in Table (2). No significant differences were detected in the concentrations of the four elements between the different seasons. Copper concentration significantly differed in autumn $(\mathrm{P}<0.05)$ and winter $(\mathrm{P}<0.01)$ between the two localities with higher concentrations in Al-Aiat area than Barrage. Iron and zinc concentrations were significantly $(\mathrm{P}<0.01)$ increased in Barrage area than Al-Aiat at spring season. Manganese concentration showed significant $(\mathrm{P}<0.001)$ higher value in Al-Aiat area than Barrage at summer season.

\section{Tissue analysis:}

The five different tissue type's liver, kidney, gills, skin and muscles were analyzed for the four metals specified and the results for both localities were summarized and tabulated (Tables 3 -6). There is a large amount of variation amongst the different tissues with regard to each metal. Therefore, the results for each metal will be explained individually.

\section{1) Effect of Location:}

\section{Iron (Table 3 and Figs. 2 \& 3):}

At winter, Fe content was significantly $(\mathrm{P}<0.05)$ decreased in the liver of female Clarias gariepinus collected from Barrage area than Al-Aiat (percentage of change: $35.5 \%$, Fig. 3). At autumn, both sexes showed significant differences between the two localities with lower and higher values of liver Fe in male (37.2 \%) and female (82.7\%) respectively collected from Barrage than Al-Aiat (Figs. 2 \& 3). In kidney, the only significant $(\mathrm{P}<0.05)$ value was showed in female fish at spring season with higher value in Barrage than Al-Aiat (53.9 \%, Fig. 3). 
Table 2: Range, mean and \pm standard deviation of iron, copper, zinc and manganese $\left(\mu \mathrm{g} \mathrm{l}^{-1}\right)$ of water samples collected seasonally from Al-Aiat and Barrage areas.

\begin{tabular}{|c|c|c|c|c|c|c|c|}
\hline \multirow{2}{*}{\multicolumn{2}{|c|}{ Season }} & \multicolumn{3}{|c|}{ Al-Aiat area (4) } & \multicolumn{3}{|c|}{ Barrage area (4) } \\
\hline & & Range & Mean & \pm S.D. & Range & Mean & \pm S.D. \\
\hline \multirow{4}{*}{ Winter } & $\mathrm{Fe}$ & $0.0421-0.0910$ & 0.0666 & 0.0282 & $0.0228-0.0697$ & 0.0463 & 0.0271 \\
\hline & $\mathrm{Cu}$ & $0.0511-0.0576$ & 0.0544 & 0.0038 & $0.0438-0.0440$ & 0.0439 & $0.0001^{\mathbf{b}}$ \\
\hline & $\mathrm{Zn}$ & $0.0032-0.0093$ & 0.0063 & 0.0035 & $0.0068-0.0089$ & 0.0079 & 0.0012 \\
\hline & $\mathrm{Mn}$ & $0.0259-0.0272$ & 0.0266 & 0.0008 & $0.0079-0.0373$ & 0.0263 & 0.0141 \\
\hline \multirow{4}{*}{ Spring } & $\mathrm{Fe}$ & $0.0182-0.0208$ & 0.0195 & 0.0015 & $0.0951-0.1656$ & 0.1304 & $0.0407^{\mathrm{b}}$ \\
\hline & $\mathrm{Cu}$ & $0.0452-0.0597$ & 0.0525 & 0.0084 & $0.0158-0.0541$ & 0.0350 & 0.0221 \\
\hline & Zn & $0.0018-0.0036$ & 0.0027 & 0.0010 & $0.0074-0.0116$ & 0.0095 & $0.0024^{\mathbf{b}}$ \\
\hline & $\mathrm{Mn}$ & $0.0229-0.0579$ & 0.0404 & 0.0202 & $0.0074-0.0294$ & 0.0212 & 0.0105 \\
\hline \multirow{4}{*}{ Summer } & $\mathrm{Fe}$ & $0.0186-0.0432$ & 0.0309 & 0.0142 & $0.0316-0.1096$ & 0.0706 & 0.0450 \\
\hline & $\mathrm{Cu}$ & $0.0447-0.0487$ & 0.0467 & 0.0023 & $0.0403-0.0666$ & 0.0535 & 0.0152 \\
\hline & Zn & $0.0001-0.0031$ & 0.0016 & 0.0017 & $0.0002-0.0092$ & 0.0047 & 0.0052 \\
\hline & $\mathrm{Mn}$ & $0.0185-0.0219$ & 0.0202 & 0.0020 & $0.0047-0.0080$ & 0.0064 & $0.0019^{c}$ \\
\hline \multirow{4}{*}{ Autumn } & $\mathrm{Fe}$ & $0.0133-0.1741$ & 0.0937 & 0.0928 & $0.0049-0.1568$ & 0.0629 & 0.0657 \\
\hline & $\mathrm{Cu}$ & $0.0457-0.0474$ & 0.0466 & 0.0010 & $0.0362-0.0440$ & 0.0401 & $0.0032^{\mathrm{a}}$ \\
\hline & $\mathrm{Zn}$ & $0.0018-0.0073$ & 0.0046 & 0.0032 & $0.0055-0.0182$ & 0.0094 & 0.0060 \\
\hline & Mn & $0.0253-0.0473$ & 0.0363 & 0.0127 & $0.0082-0.0272$ & 0.0201 & 0.0091 \\
\hline \multirow{4}{*}{ ANOVA } & $\mathrm{Fe}$ & \multirow{4}{*}{\multicolumn{3}{|c|}{$\begin{array}{l}\text { N.S. } \\
\text { N.S. } \\
\text { N.S. } \\
\text { N.S. }\end{array}$}} & \multirow{4}{*}{\multicolumn{3}{|c|}{$\begin{array}{l}\text { N.S. } \\
\text { N.S. } \\
\text { N.S. } \\
\text { N.S. }\end{array}$}} \\
\hline & $\mathrm{Cu}$ & & & & & & \\
\hline & $\mathrm{Zn}$ & & & & & & \\
\hline & $\mathrm{Mn}$ & & & & & & \\
\hline
\end{tabular}

Table 3: Mean \pm standard deviation of iron concentration ( $\mu \mathrm{g} \mathrm{g}^{-1}$ dry weight) in different tissues of both sexes of Clarias gariepinus collected from Al-Aiat (site I) and Barrage (site II) areas during the four seasons.

\begin{tabular}{|c|c|c|c|c|c|c|c|c|c|c|c|}
\hline \multirow{2}{*}{ Tissue } & \multirow{2}{*}{ Site } & \multicolumn{2}{|c|}{ Winter } & \multicolumn{2}{|c|}{ Spring } & \multicolumn{2}{|c|}{ Summer } & \multicolumn{2}{|c|}{ Autumn } & \multicolumn{2}{|c|}{ ANOVA } \\
\hline & & Male & Female & Male & Female & Male & Female & Male & Female & Male & Female \\
\hline \multirow{2}{*}{ Liver } & I & $\begin{array}{c}943.4 \pm \\
328.0\end{array}$ & $\begin{array}{c}1353.1 \pm \\
374.6^{*}\end{array}$ & $\begin{array}{c}355.8 \pm \\
145.8\end{array}$ & $\begin{array}{c}296.2 \pm \\
103.5\end{array}$ & $\begin{array}{c}402.5 \pm \\
201.6\end{array}$ & $\begin{array}{c}642.6 \pm \\
320.7\end{array}$ & $\begin{array}{c}956.2 \pm \\
364.5\end{array}$ & $\begin{array}{c}578.9 \pm \\
277.6^{*}\end{array}$ & $\mathrm{P}<0.01$ & $\mathrm{P}<0.01$ \\
\hline & II & $\begin{array}{c}979.5 \pm \\
397.0\end{array}$ & $\begin{array}{c}872.3 \pm \\
415.3^{\mathrm{a}}\end{array}$ & $\begin{array}{c}309.5 \pm \\
177.3\end{array}$ & $\begin{array}{c}434.7 \pm \\
195.5\end{array}$ & $\begin{array}{c}483.8 \pm \\
205.6\end{array}$ & $\begin{array}{c}470.3 \pm \\
220.0\end{array}$ & $\begin{array}{c}600.3 \pm \\
298.1^{\mathrm{a}}\end{array}$ & $\begin{array}{c}1057.5 \pm \\
408.3^{* \mathbf{b}}\end{array}$ & $\mathrm{P}<0.05$ & $\mathrm{P}<0.05$ \\
\hline \multirow{2}{*}{ Kidney } & I & $\begin{array}{c}904.1 \pm \\
333.9\end{array}$ & $\begin{array}{c}681.5 \pm \\
86.3\end{array}$ & $\begin{array}{c}392.3 \pm \\
101.2\end{array}$ & $\begin{array}{c}413.7 \pm \\
45.7\end{array}$ & $\begin{array}{c}416.2 \pm \\
98.8\end{array}$ & $\begin{array}{c}530.3 \pm \\
86.7^{*}\end{array}$ & $\begin{array}{c}502.7 \pm \\
118.5\end{array}$ & $\begin{array}{l}773.9 \pm \\
208.4^{* *}\end{array}$ & $\mathrm{P}<0.01$ & $\mathrm{P}<0.05$ \\
\hline & II & $\begin{array}{c}702.6 \pm \\
238.0\end{array}$ & $\begin{array}{c}596.6 \pm \\
140.6\end{array}$ & $\begin{array}{c}417.7 \pm \\
100.1\end{array}$ & $\begin{array}{l}636.6 \pm \\
238.8^{* a}\end{array}$ & $\begin{array}{c}464.5 \pm \\
99.3\end{array}$ & $\begin{array}{c}519.2 \pm \\
158.3\end{array}$ & $\begin{array}{c}597.7 \pm \\
266.4\end{array}$ & $\begin{array}{c}752.4 \pm \\
201.2\end{array}$ & N.S. & N.S. \\
\hline \multirow{2}{*}{ Gills } & I & $\begin{array}{c}386.1 \pm \\
140.7\end{array}$ & $\begin{array}{c}345.2 \pm \\
93.6\end{array}$ & $\begin{array}{c}470.3 \pm \\
92.9\end{array}$ & $\begin{array}{c}461.5 \pm \\
74.1\end{array}$ & $\begin{array}{c}404.4 \pm \\
155.5\end{array}$ & $\begin{array}{c}352.5 \pm \\
157.6\end{array}$ & $\begin{array}{c}401.7 \pm \\
56.8\end{array}$ & $\begin{array}{c}504.5 \pm \\
195.4\end{array}$ & N.S. & N.S. \\
\hline & II & $\begin{array}{c}354.3 \pm \\
50.3\end{array}$ & $\begin{array}{c}413.3 \pm \\
187.1\end{array}$ & $\begin{array}{c}413.7 \pm \\
150.8\end{array}$ & $\begin{array}{c}410.3 \pm \\
104.5\end{array}$ & $\begin{array}{c}354.6 \pm \\
93.6\end{array}$ & $\begin{array}{c}357.0 \pm \\
146.9\end{array}$ & $\begin{array}{c}316.4 \pm \\
103.0^{\mathbf{a}}\end{array}$ & $\begin{array}{l}208.0 \pm \\
103.5^{* \mathbf{b}}\end{array}$ & N.S. & N.S. \\
\hline \multirow{2}{*}{ Skin } & I & $\begin{array}{c}234.6 \pm \\
81.0\end{array}$ & $\begin{array}{c}219.3 \pm \\
26.0\end{array}$ & $\begin{array}{c}209.6 \pm \\
97.5\end{array}$ & $\begin{array}{c}247.1 \pm \\
113.6\end{array}$ & $\begin{array}{c}365.3 \pm \\
150.0\end{array}$ & $\begin{array}{c}408.7 \pm \\
207.4\end{array}$ & $\begin{array}{c}439.5 \pm \\
200.4\end{array}$ & $\begin{array}{c}354.6 \pm \\
160.6\end{array}$ & $\mathrm{P}<0.05$ & N.S. \\
\hline & II & $\begin{array}{c}309.8 \pm \\
62.9^{\mathrm{a}}\end{array}$ & $\begin{array}{c}316.0 \pm \\
91.0^{\mathbf{b}}\end{array}$ & $\begin{array}{c}282.7 \pm \\
60.9\end{array}$ & $\begin{array}{l}419.6 \pm \\
164.2^{* a}\end{array}$ & $\begin{array}{c}366.3 \pm \\
81.8\end{array}$ & $\begin{array}{c}461.1 \pm \\
138.0\end{array}$ & $\begin{array}{c}292.7 \pm \\
115.6^{\mathbf{a}}\end{array}$ & $\begin{array}{c}270.6 \pm \\
63.6\end{array}$ & N.S. & N.S. \\
\hline \multirow{2}{*}{ Muscles } & I & $\begin{array}{c}105.5 \pm \\
37.8\end{array}$ & $\begin{array}{c}118.1 \pm \\
40.5\end{array}$ & $\begin{array}{c}86.7 \pm \\
43.0\end{array}$ & $\begin{array}{c}68.5 \pm \\
33.1\end{array}$ & $\begin{array}{c}104.7 \pm \\
23.7\end{array}$ & $\begin{array}{c}96.3 \pm \\
10.7\end{array}$ & $\begin{array}{c}113.7 \pm \\
26.4\end{array}$ & $\begin{array}{l}85.7 \pm \\
13.9^{* *}\end{array}$ & N.S. & N.S. \\
\hline & II & $\begin{array}{c}193.4 \pm \\
93.2^{\mathbf{b}}\end{array}$ & $\begin{array}{c}159.6 \pm \\
43.7\end{array}$ & $\begin{array}{c}93.0 \pm \\
28.9\end{array}$ & $\begin{array}{c}93.6 \pm \\
8.5\end{array}$ & $\begin{array}{c}103.1 \pm \\
28.5\end{array}$ & $\begin{array}{c}117.3 \pm \\
57.9\end{array}$ & $\begin{array}{c}87.5 \pm \\
24.1^{\mathrm{a}}\end{array}$ & $\begin{array}{c}116.9 \pm \\
13.8^{* * *} \mathrm{c}\end{array}$ & $\mathrm{P}<0.05$ & N.S. \\
\hline
\end{tabular}

Significant difference between the two sexes in the same site are represented by: *: $\mathrm{P}<0.05$; **; $\mathrm{P}<0.01$ and $* * *: \mathrm{P}<0.001$

Significant difference between the two sites in the same sex are represented by: a: $\mathrm{P}<0.05$, b: $\mathrm{P}<0.01$ and c: $\mathrm{P}<0.001$

Numbers of samples in each site are represented in table 1 


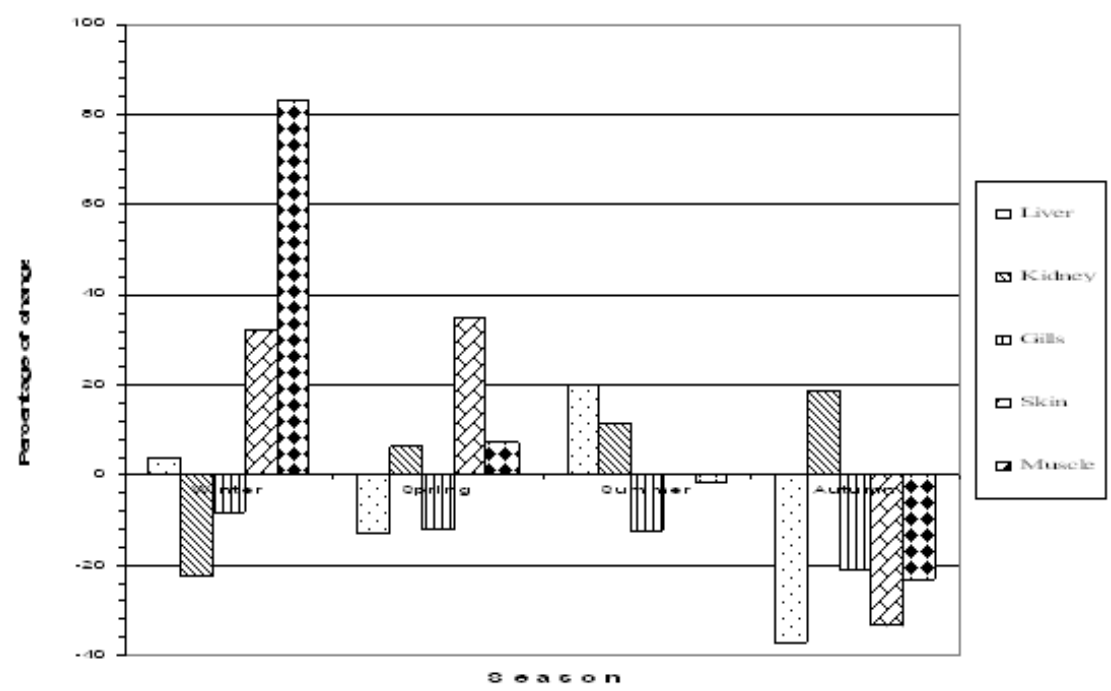

Fig. 2: The percentage of change in the iron concentrations in the liver, kidney, gills skin and muscles of male Clarias gariepinus from Barrage (site II) relative to Al-Aiat (site I) during the four seasons.

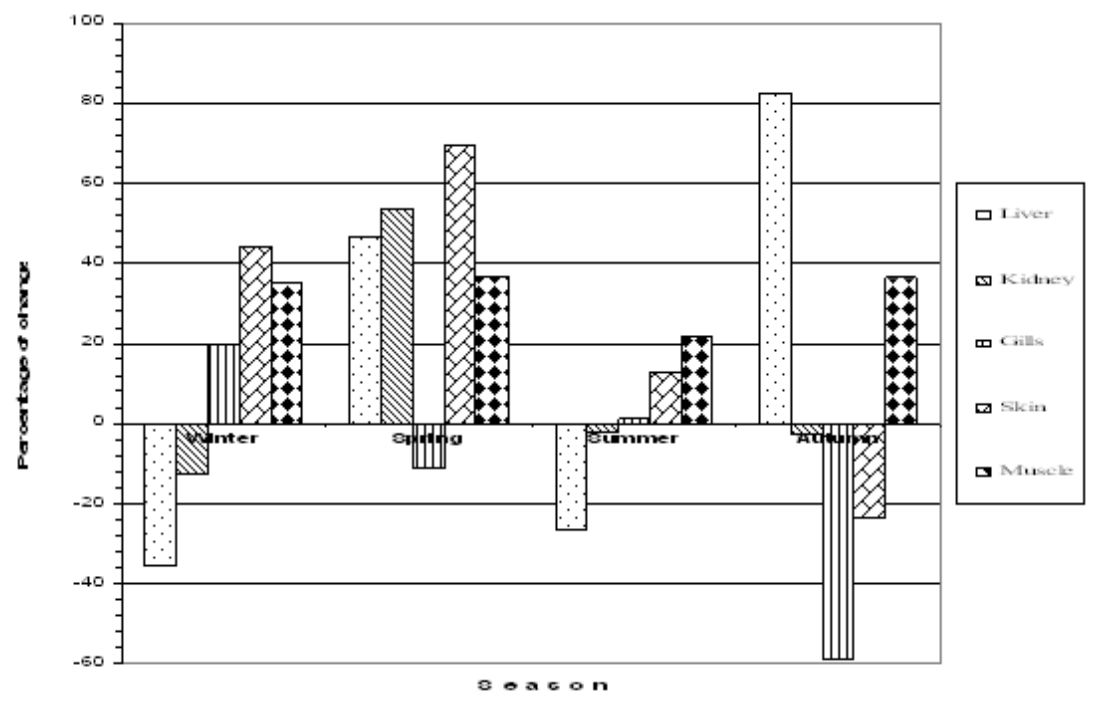

Fig. 3: The percentage of change in the iron concentrations in the liver, kidney, gills skin and muscles of female Clarias gariepinus from Barrage (site II) relative to Al-Aiat (site I) during the four seasons.

Iron content in gills of both sexes at autumn season showed significant lower values in Barrage than Al-Aiat (21.2 and $58.8 \%$ for $\delta^{\lambda}$ and $q$ respectively). In skin, Fe content in both sexes at winter and female fish at spring showed significant higher values in Barrage area than Al-Aiat (32.1; 44.1 and $69.8 \%$ respectively), while male fish at autumn recorded significant $(\mathrm{P}<0.05)$ lower value $(33.4 \%)$. Iron content in muscles at winter showed significant $(\mathrm{P}<0.01)$ higher value in male fish from Barrage than Al-Aiat (83.2 \%). At autumn, significant lower (23.1\%) and higher $(36.4 \%)$ values were detected in males and females respectively between the two localities.

\section{Copper (Table 4 and Figs. 4 \& 5):}

Comparison between the two areas showed that the liver copper of fish collected from Barrage area significantly differed than that from Al-Aiat area as follows: lower at winter for females (percentage of change: $61.5 \%$, Fig. 5) and at autumn for males 
(44.8 \%, Fig. 4) and higher values at summer for both sexes (64.7 and $103.4 \%$ for $\hat{\sigma}$ and $q$ respectively). Both sexes from Barrage area recorded lower (37.4 and $48.4 \%$ for $\hat{\sigma}$ and $q$ respectively) and higher (98.1 and $47.7 \%$ for $\hat{\delta}$ and $q$ respectively) significant values of $\mathrm{Cu}$ in the kidney at winter and summer respectively than that from Al-Aiat area. Gills Cu from both sexes at winter (93.0 and $122.7 \%$ for ${ }^{\lambda}$ and $q$ respectively); females at spring (31.9\%) and males at autumn (27.5\%) from Barrage area recorded significant higher values than that from Al-Aiat. The same significant higher values were demonstrated in the skin $\mathrm{Cu}$ from females at winter (367.5\%); both sexes at spring (903.8 and $518.2 \%$ for and $q$ respectively) and males at autumn (229.5\%). Also, the significant higher values were detected in the muscles $\mathrm{Cu}$ from males at summer (103.3 \%) and both sexes at autumn (40.0 and $50.3 \%$ for and $q$ respectively).

Table 4: Mean \pm standard deviation of copper concentration ( $\mu \mathrm{g} \mathrm{g}^{-1}$ dry weight) in different tissues of both sexes of Clarias gariepinus collected from Al-Aiat (site I) and Barrage (site II) areas during the four seasons.

\begin{tabular}{|c|c|c|c|c|c|c|c|c|c|c|c|}
\hline \multirow{2}{*}{ Tissue } & \multirow{2}{*}{ Site } & \multicolumn{2}{|c|}{ Winter } & \multicolumn{2}{|c|}{ Spring } & \multicolumn{2}{|c|}{ Summer } & \multicolumn{2}{|c|}{ Autumn } & \multicolumn{2}{|c|}{ ANOVA } \\
\hline & & Male & Female & Male & Female & Male & Female & Male & Female & Male & Female \\
\hline \multirow{2}{*}{ Liver } & I & $\begin{array}{c}226.0 \pm \\
97.5\end{array}$ & $\begin{array}{l}394.7 \pm \\
110.3^{* *}\end{array}$ & $\begin{array}{c}123.3 \pm \\
79.6\end{array}$ & $\begin{array}{l}230.9 \pm \\
110.1^{*}\end{array}$ & $\begin{array}{c}165.8 \pm \\
61.9\end{array}$ & $\begin{array}{c}161.8 \pm \\
70.7\end{array}$ & $\begin{array}{c}257.5 \pm \\
134.9\end{array}$ & $\begin{array}{c}213.3 \pm \\
107.0\end{array}$ & N.S. & $\mathrm{P}<0.05$ \\
\hline & II & $\begin{array}{c}253.7 \pm \\
167.2 \\
\end{array}$ & $\begin{array}{c}152.2 \pm \\
60.6^{\mathbf{c}} \\
\end{array}$ & $\begin{array}{c}124.1 \pm \\
49.9 \\
\end{array}$ & $\begin{array}{c}153.7 \pm \\
68.5 \\
\end{array}$ & $\begin{array}{c}273.0 \pm \\
34.7^{\mathrm{c}} \\
\end{array}$ & $\begin{array}{r}328.9 \pm \\
168.9^{\mathrm{a}} \\
\end{array}$ & $\begin{array}{c}142.2 \pm \\
74.2^{\mathrm{a}} \\
\end{array}$ & $\begin{array}{c}284.8 \pm \\
143.2^{*} \\
\end{array}$ & N.S. & N.S. \\
\hline \multirow{2}{*}{ Kidney } & I & $\begin{array}{c}67.631 \pm \\
31.860\end{array}$ & $\begin{array}{c}80.141 \pm \\
12.491\end{array}$ & $\begin{array}{c}29.639 \pm \\
11.989\end{array}$ & $\begin{array}{c}29.049 \pm \\
8.986\end{array}$ & $\begin{array}{c}38.269 \pm \\
12.923\end{array}$ & $\begin{array}{l}57.487 \pm \\
14.429^{* *}\end{array}$ & $\begin{array}{c}77.490 \pm \\
30.615\end{array}$ & $\begin{array}{c}98.709 \pm \\
36.386\end{array}$ & $\mathrm{P}<0.05$ & $\mathrm{P}<0.01$ \\
\hline & II & $\begin{array}{r}42.345 \pm \\
18.015^{\mathrm{a}} \\
\end{array}$ & $\begin{array}{c}41.353 \pm \\
16.059^{c}\end{array}$ & $\begin{array}{c}30.741 \pm \\
11.087 \\
\end{array}$ & $\begin{array}{c}31.176 \pm \\
12.472 \\
\end{array}$ & $\begin{array}{c}75.789 \pm \\
23.044^{\mathrm{c}} \\
\end{array}$ & $\begin{array}{c}84.890 \pm \\
21.660^{\mathbf{b}}\end{array}$ & $\begin{array}{c}85.011 \pm \\
28.468 \\
\end{array}$ & $\begin{array}{c}97.231 \pm \\
45.248 \\
\end{array}$ & $\mathrm{P}<0.05$ & $\mathrm{P}<0.01$ \\
\hline \multirow{2}{*}{ Gills } & I & $\begin{array}{c}19.465 \pm \\
5.896\end{array}$ & $\begin{array}{c}24.972 \pm \\
7.165\end{array}$ & $\begin{array}{c}27.010 \pm \\
5.659\end{array}$ & $\begin{array}{c}19.449 \pm \\
4.097^{* *}\end{array}$ & $\begin{array}{c}21.888 \pm \\
7.912\end{array}$ & $\begin{array}{c}19.178 \pm \\
4.670\end{array}$ & $\begin{array}{c}17.718 \pm \\
4.023\end{array}$ & $\begin{array}{l}27.489 \pm \\
10.498^{* *}\end{array}$ & N.S. & N.S. \\
\hline & II & $\begin{array}{c}37.564 \pm \\
13.495^{c} \\
\end{array}$ & $\begin{array}{c}55.604 \pm \\
24.894^{\mathbf{b}} \\
\end{array}$ & $\begin{array}{c}23.194 \pm \\
11.554 \\
\end{array}$ & $\begin{array}{c}25.659 \pm \\
2.162^{\mathbf{b}} \\
\end{array}$ & $\begin{array}{c}20.614 \pm \\
2.925 \\
\end{array}$ & $\begin{array}{c}19.909 \pm \\
10.083 \\
\end{array}$ & $\begin{array}{c}22.591 \pm \\
4.736^{\mathbf{a}} \\
\end{array}$ & $\begin{array}{c}22.706 \pm \\
11.385 \\
\end{array}$ & $\mathrm{P}<0.05$ & $\mathrm{P}<0.05$ \\
\hline \multirow{2}{*}{ Skin } & I & $\begin{array}{c}1.127 \pm \\
0.550\end{array}$ & $\begin{array}{l}0.428 \pm \\
0.200^{* *}\end{array}$ & $\begin{array}{c}0.577 \pm \\
0.285\end{array}$ & $\begin{array}{c}1.206 \pm \\
0.663^{*}\end{array}$ & $\begin{array}{c}4.933 \pm \\
2.521\end{array}$ & $\begin{array}{c}4.742 \pm \\
2.252\end{array}$ & $\begin{array}{c}2.454 \pm \\
1.255\end{array}$ & $\begin{array}{c}4.684 \pm \\
2.339^{*}\end{array}$ & $\mathrm{P}<0.01$ & $\mathrm{P}<0.01$ \\
\hline & II & $\begin{array}{c}1.300 \pm \\
0.697\end{array}$ & $\begin{array}{c}2.001 \pm \\
1.022^{\mathrm{c}}\end{array}$ & $\begin{array}{l}5.790 \pm \\
2.483^{\mathrm{c}} \\
\end{array}$ & $\begin{array}{l}7.458 \pm \\
3.069^{c} \\
\end{array}$ & $\begin{array}{c}5.299 \pm \\
2.662 \\
\end{array}$ & $\begin{array}{c}7.777 \pm \\
4.049 \\
\end{array}$ & $\begin{array}{r}8.085 \pm \\
4.161^{\mathrm{c}} \\
\end{array}$ & $\begin{array}{c}7.605 \pm \\
3.896 \\
\end{array}$ & $\mathrm{P}<0.05$ & $\mathrm{P}<0.05$ \\
\hline \multirow{2}{*}{ Muscles } & I & $\begin{array}{c}2.831 \pm \\
1.477\end{array}$ & $\begin{array}{c}2.575 \pm \\
1.103\end{array}$ & $\begin{array}{c}2.541 \pm \\
0.706\end{array}$ & $\begin{array}{l}3.789 \pm \\
1.404^{*}\end{array}$ & $\begin{array}{c}4.250 \pm \\
1.356\end{array}$ & $\begin{array}{c}4.341 \pm \\
1.395\end{array}$ & $\begin{array}{c}6.577 \pm \\
1.304\end{array}$ & $\begin{array}{c}5.706 \pm \\
1.745\end{array}$ & $\mathrm{P}<0.01$ & $\mathrm{P}<0.05$ \\
\hline & II & $\begin{array}{c}2.202 \pm \\
1.140\end{array}$ & $\begin{array}{c}1.890 \pm \\
0.894\end{array}$ & $\begin{array}{c}3.280 \pm \\
1.266\end{array}$ & $\begin{array}{c}3.135 \pm \\
1.456\end{array}$ & $\begin{array}{l}8.642 \pm \\
4.364^{b}\end{array}$ & $\begin{array}{c}5.798 \pm \\
2.395\end{array}$ & $\begin{array}{l}9.205 \pm \\
2.559^{\mathbf{b}}\end{array}$ & $\begin{array}{c}8.577 \pm \\
3.367^{\mathrm{a}}\end{array}$ & $\mathrm{P}<0.01$ & $\mathrm{P}<0.01$ \\
\hline
\end{tabular}

See footnote table 3

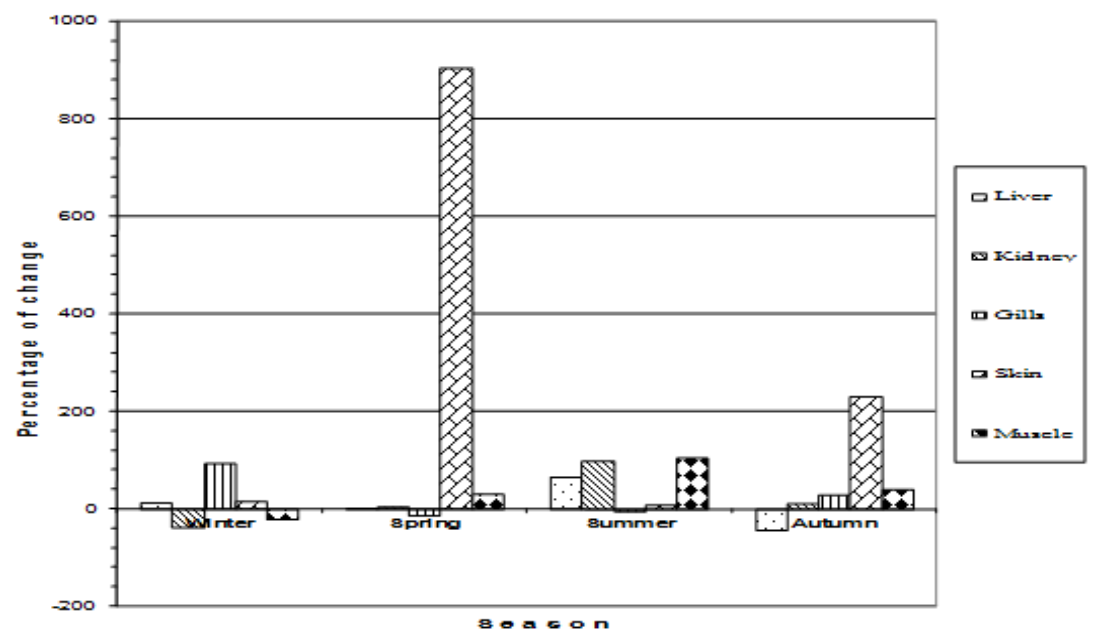

Fig. 4: The percentage of change in the copper concentrations in the liver, kidney, gills skin and muscles of male Clarias gariepinus from Barrage (site II) relative to Al-Aiat (site I) during the four seasons. 


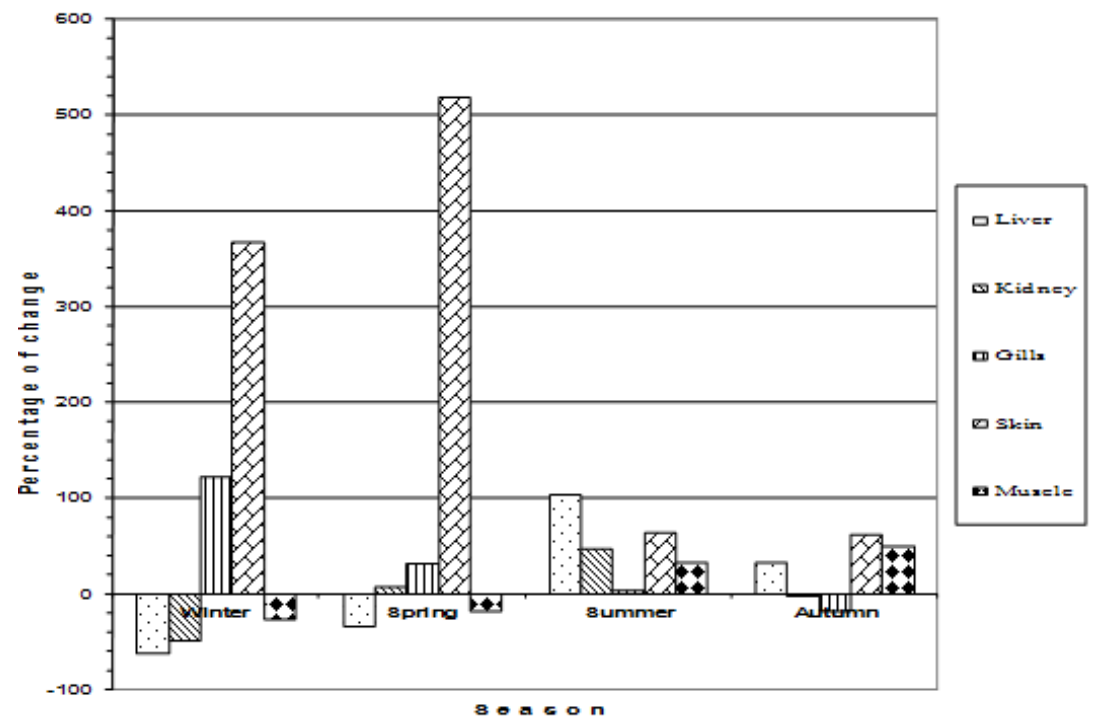

Fig. 5: The percentage of change in the copper concentrations in the liver, kidney, gills skin and muscles of female Clarias gariepinus from Barrage (site II) relative to Al-Aiat (site I) during the four seasons.

\section{Zinc (Table 5 and Figs. 6 \& 7):}

At winter, both sexes of fish collected from Barrage area recorded significant lower values (percentage change: 26.4 and $39.8 \%$ for $\delta$ and $\rho$ respectively) of the liver $\mathrm{Zn}$ than that from Al-Aiat area. Contrary at summer, both sexes recorded significant higher values of $\mathrm{Zn}$ in both liver (58.4 and $66.6 \%$ for $\delta$ and $q$ respectively) and skin (71.7 and $51.8 \%$ for $\delta$ and $q$ respectively). Also, $\mathrm{Zn}$ in the gills of male fish recorded a significant higher value at autumn (24.7\%).

Table 5: Mean \pm standard deviation of zinc concentration ( $\mu \mathrm{g} \mathrm{g}^{-1}$ dry weight) in different tissues of both sexes of Clarias gariepinus collected from Al-Aiat (site I) and Barrage (site II) areas during the four seasons.

\begin{tabular}{|c|c|c|c|c|c|c|c|c|c|c|c|}
\hline \multirow{2}{*}{ Tissue } & \multirow{2}{*}{ Site } & \multicolumn{2}{|c|}{ Winter } & \multicolumn{2}{|c|}{ Spring } & \multicolumn{2}{|c|}{ Summer } & \multicolumn{2}{|c|}{ Autumn } & \multicolumn{2}{|c|}{ ANOVA } \\
\hline & & Male & Female & Male & Female & Male & Female & Male & Female & Male & Female \\
\hline \multirow{2}{*}{ Liver } & I & $\begin{array}{c}197.0 \pm \\
54.5\end{array}$ & $\begin{array}{c}201.0 \pm \\
35.9\end{array}$ & $\begin{array}{c}139.2 \pm \\
49.0\end{array}$ & $\begin{array}{c}136.5 \pm \\
57.2\end{array}$ & $\begin{array}{c}138.9 \pm \\
28.0\end{array}$ & $\begin{array}{c}135.4 \pm \\
30.2\end{array}$ & $\begin{array}{c}165.7 \pm \\
61.3\end{array}$ & $\begin{array}{c}182.4 \pm \\
53.3\end{array}$ & N.S. & N.S. \\
\hline & II & $\begin{array}{c}144.9 \pm \\
33.8^{\mathbf{a}}\end{array}$ & $\begin{array}{c}121.0^{ \pm} \\
61.8^{\mathbf{b}}\end{array}$ & $\begin{array}{c}115.7 \pm \\
21.3\end{array}$ & $\begin{array}{c}125.6 \pm \\
28.5\end{array}$ & $\begin{array}{l}220.1 \pm \\
68.5^{\mathbf{b}}\end{array}$ & $\begin{array}{c}225.5 \pm \\
71.6^{\mathbf{b}}\end{array}$ & $\begin{array}{c}175.4 \pm \\
44.2\end{array}$ & $\begin{array}{c}199.9 \pm \\
41.2\end{array}$ & $\begin{array}{l}\mathrm{P}< \\
0.05\end{array}$ & $\mathrm{P}<0.05$ \\
\hline \multirow{2}{*}{ Kidney } & I & $\begin{array}{c}238.0 \pm \\
97.2\end{array}$ & $\begin{array}{c}290.3 \pm \\
67.6\end{array}$ & $\begin{array}{c}133.3 \pm \\
22.4\end{array}$ & $\begin{array}{c}134.9 \pm \\
19.8\end{array}$ & $\begin{array}{c}167.7 \pm \\
45.9\end{array}$ & $\begin{array}{l}234.4 \pm \\
37.1^{* *}\end{array}$ & $\begin{array}{c}158.6 \pm \\
19.0\end{array}$ & $\begin{array}{c}163.0 \pm \\
53.2\end{array}$ & $\begin{array}{c}\mathrm{P}< \\
0.05\end{array}$ & $\mathrm{P}<0.01$ \\
\hline & II & $\begin{array}{c}226.9 \pm \\
48.4 \\
\end{array}$ & $\begin{array}{c}245.3 \pm \\
67.7 \\
\end{array}$ & $\begin{array}{c}141.3 \pm \\
22.4 \\
\end{array}$ & $\begin{array}{c}144.0 \pm \\
24.9 \\
\end{array}$ & $\begin{array}{c}174.0 \pm \\
35.6 \\
\end{array}$ & $\begin{array}{c}260.0 \pm \\
128.1 \\
\end{array}$ & $\begin{array}{c}171.4 \pm \\
46.0 \\
\end{array}$ & $\begin{array}{c}147.5 \pm \\
37.9 \\
\end{array}$ & $\begin{array}{l}\mathrm{P}< \\
0.05\end{array}$ & N.S. \\
\hline \multirow{2}{*}{ Gills } & I & $\begin{array}{c}74.786 \pm \\
5.350\end{array}$ & $\begin{array}{c}72.434 \pm \\
6.972\end{array}$ & $\begin{array}{c}72.056 \pm \\
9.220\end{array}$ & $\begin{array}{c}72.756 \pm \\
7.345\end{array}$ & $\begin{array}{c}80.173 \pm \\
18.442\end{array}$ & $\begin{array}{c}77.456 \pm \\
9.617\end{array}$ & $\begin{array}{c}65.425 \pm \\
15.604\end{array}$ & $\begin{array}{c}76.726 \pm \\
32.236\end{array}$ & N.S. & N.S. \\
\hline & II & $\begin{array}{c}69.786 \pm \\
19.404\end{array}$ & $\begin{array}{c}83.381 \pm \\
18.084\end{array}$ & $\begin{array}{c}72.904 \pm \\
14.756\end{array}$ & $\begin{array}{c}70.035 \pm \\
13.350\end{array}$ & $\begin{array}{c}68.897 \pm \\
13.184\end{array}$ & $\begin{array}{c}79.839 \pm \\
26.136\end{array}$ & $\begin{array}{c}81.569 \pm \\
12.080^{\mathrm{a}}\end{array}$ & $\begin{array}{c}75.291 \pm \\
8.352\end{array}$ & N.S. & N.S. \\
\hline \multirow{2}{*}{ Skin } & I & $\begin{array}{c}60.787 \pm \\
21.527\end{array}$ & $\begin{array}{c}52.459 \pm \\
11.990\end{array}$ & $\begin{array}{c}53.683 \pm \\
23.534\end{array}$ & $\begin{array}{c}48.790 \pm \\
16.624\end{array}$ & $\begin{array}{c}51.154 \pm \\
22.691\end{array}$ & $\begin{array}{l}88.525 \pm \\
22.586^{* *}\end{array}$ & $\begin{array}{c}76.928 \pm \\
8.738\end{array}$ & $\begin{array}{c}103.286 \pm \\
31.219^{*}\end{array}$ & N.S. & $\mathrm{P}<0.05$ \\
\hline & II & $\begin{array}{c}41.246 \pm \\
23.562\end{array}$ & $\begin{array}{c}42.901 \pm \\
18.151\end{array}$ & $\begin{array}{c}41.718 \pm \\
11.726\end{array}$ & $\begin{array}{c}51.586 \pm \\
17.299\end{array}$ & $\begin{array}{c}87.846 \pm \\
41.681^{\mathrm{a}}\end{array}$ & $\begin{array}{c}134.417 \pm \\
10.704^{* *_{c}}\end{array}$ & $\begin{array}{c}87.088 \pm \\
19.311\end{array}$ & $\begin{array}{c}87.250 \pm \\
17.162\end{array}$ & $\begin{array}{l}\mathrm{P}< \\
0.05\end{array}$ & $\mathrm{P}<0.01$ \\
\hline \multirow{2}{*}{ Muscles } & I & $\begin{array}{c}26.681 \pm \\
11.100\end{array}$ & $\begin{array}{c}30.688 \pm \\
8.732\end{array}$ & $\begin{array}{c}27.487 \pm \\
10.106\end{array}$ & $\begin{array}{c}34.473 \pm \\
17.288\end{array}$ & $\begin{array}{c}40.532 \pm \\
7.837\end{array}$ & $\begin{array}{l}25.367 \pm \\
12.866^{* *}\end{array}$ & $\begin{array}{c}27.557 \pm \\
13.452\end{array}$ & $\begin{array}{c}26.184 \pm \\
13.455\end{array}$ & N.S. & N.S. \\
\hline & II & $\begin{array}{c}25.914 \pm \\
8.635\end{array}$ & $\begin{array}{c}27.479 \pm \\
11.946\end{array}$ & $\begin{array}{c}28.109 \pm \\
14.041\end{array}$ & $\begin{array}{c}39.346 \pm \\
9.310\end{array}$ & $\begin{array}{c}52.573 \pm \\
27.456\end{array}$ & $\begin{array}{c}37.780 \pm \\
16.530\end{array}$ & $\begin{array}{c}28.072 \pm \\
14.564\end{array}$ & $\begin{array}{c}33.463 \pm \\
12.797\end{array}$ & N.S. & N.S. \\
\hline
\end{tabular}

See footnote table 3 


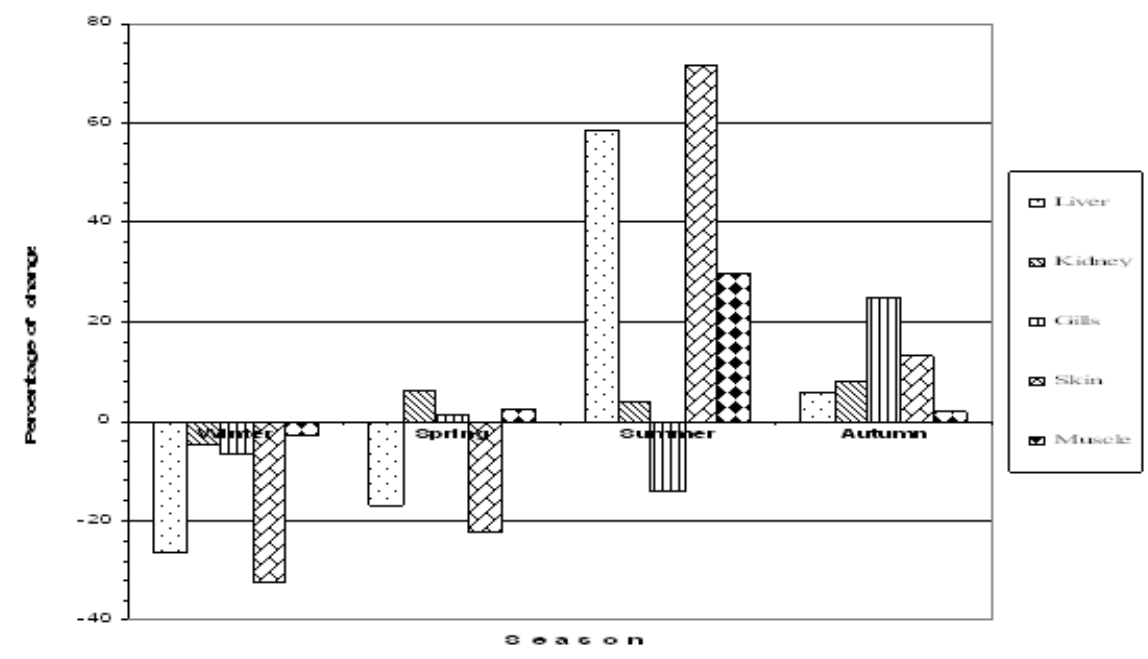

Fig. 6: The percentage of change in the zinc concentrations in the liver, kidney, gills skin and muscles of male Clarias gariepinus from Barrage (site II) relative to Al-Aiat (site I) during the four seasons.

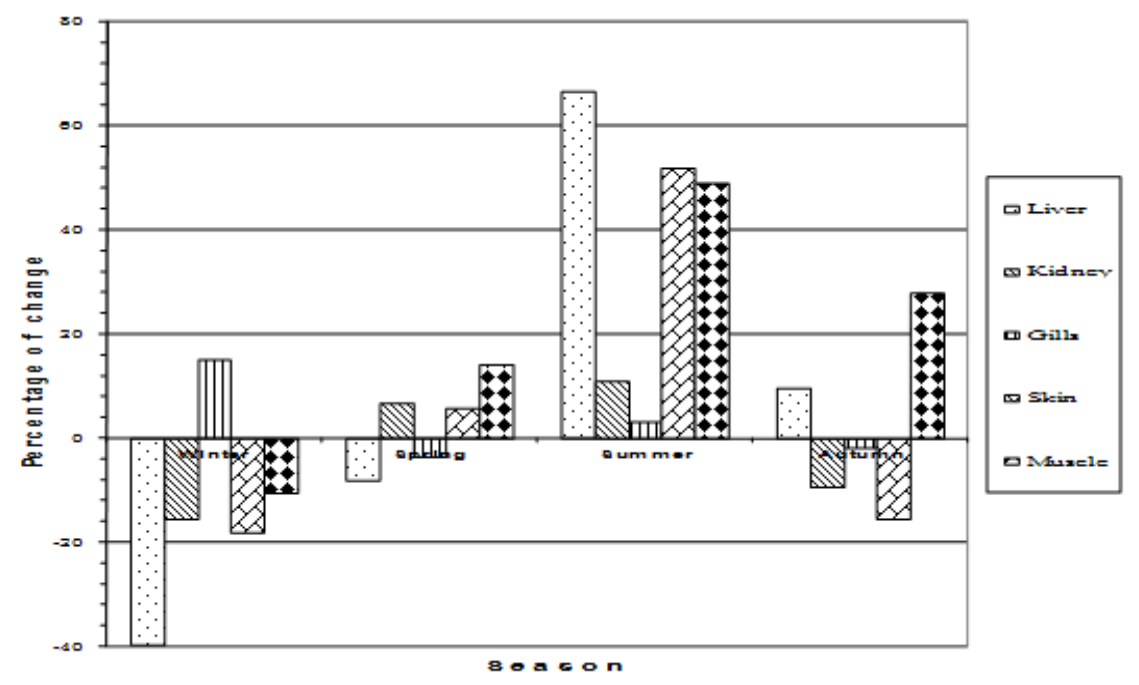

Fig. 7: The percentage of change in the zinc concentrations in the liver, kidney, gills skin and muscles of female Clarias gariepinus from Barrage (site II) relative to Al-Aiat (site I) during the four seasons.

\section{Manganese (Table 6 and Figs. 8 \& 9):}

At winter, both sexes of fish collected from Barrage area showed significant lower values of $\mathrm{Mn}$ in the kidney (percentage change: 38.8 and $31.3 \%$ for $\hat{\delta}$ and $q$ respectively); skin (42.1 and $34.4 \%$ for $\partial$ and $q$ respectively) and muscles (60.3 and $57.5 \%$ for $\delta$ and $O$ respectively) than that from Al-Aiat area. In the same season, females recorded a significant lower value of the liver Mn (36.5\%), while males showed significant higher values of $\mathrm{Mn}$ in both liver (80.6 \%) and gills (107.6 \%). At spring, the only significant lower value was detected in the gills of males (49.3\%) collected from Barrage area than that from Al-Aiat area. At summer, significant higher values of the Mn contents in the five organs of males (176.3, 64.1, 129.8, 71.0 and $53.6 \%$ for liver, kidney, gills, skin and muscles respectively) and only in the gills of females (247.2 \%) from Barrage area were demonstrated than that from Al-Aiat. At autumn, significant lower values of Mn were recorded in the kidney (57.8 and $62.1 \%$ 
for $\widehat{\delta}$ and $q$ respectively) and skin (55.6 and $60.1 \%$ for $\widehat{\partial}$ and $q$ respectively) and in the muscles of female fish (49.1\%) collected from Barrage than Al-Aiat area.

Table 6: Mean \pm standard deviation of manganese concentration ( $\mu \mathrm{g} \mathrm{g}^{-1}$ dry weight) in different tissues of both sexes of Clarias gariepinus collected from Al-Aiat (site I) and Barrage (site II) areas during the four seasons.

\begin{tabular}{|c|c|c|c|c|c|c|c|c|c|c|c|}
\hline \multirow{2}{*}{ Tissue } & \multirow{2}{*}{ Site } & \multicolumn{2}{|c|}{ Winter } & \multicolumn{2}{|c|}{ Spring } & \multicolumn{2}{|c|}{ Summer } & \multicolumn{2}{|c|}{ Autumn } & \multicolumn{2}{|c|}{ ANOVA } \\
\hline & & Male & Female & Male & Female & Male & Female & Male & Female & Male & Female \\
\hline \multirow{2}{*}{ Liver } & I & $\begin{array}{c}1.528 \pm \\
0.923\end{array}$ & $\begin{array}{l}3.567 \pm \\
0.569^{* * *}\end{array}$ & $\begin{array}{c}2.485 \pm \\
1.129\end{array}$ & $\begin{array}{c}3.766 \pm \\
1.382\end{array}$ & $\begin{array}{c}1.786 \pm \\
0.829\end{array}$ & $\begin{array}{l}3.102 \pm \\
0.697^{* *}\end{array}$ & $\begin{array}{c}4.301 \pm \\
1.570\end{array}$ & $\begin{array}{c}4.612 \pm \\
0.947\end{array}$ & $\mathrm{P}<0.01$ & N.S. \\
\hline & II & $\begin{array}{c}2.760 \pm \\
1.082^{\mathrm{a}}\end{array}$ & $\begin{array}{l}2.266 \pm \\
1.112^{\mathrm{b}}\end{array}$ & $\begin{array}{c}1.617 \pm \\
0.874\end{array}$ & $\begin{array}{l}3.938 \pm \\
1.181^{* * *}\end{array}$ & $\begin{array}{l}4.936 \pm \\
2.523^{c}\end{array}$ & $\begin{array}{c}4.350 \pm \\
2.251\end{array}$ & $\begin{array}{c}4.383 \pm \\
1.896\end{array}$ & $\begin{array}{c}5.104 \pm \\
1.110\end{array}$ & $\mathrm{P}<0.05$ & $P<0.05$ \\
\hline \multirow{2}{*}{ Kidney } & I & $\begin{array}{c}11.192 \pm \\
3.960\end{array}$ & $\begin{array}{c}10.651 \pm \\
0.934\end{array}$ & $\begin{array}{c}14.370 \pm \\
6.243\end{array}$ & $\begin{array}{c}11.682 \pm \\
5.876\end{array}$ & $\begin{array}{c}24.654 \pm \\
5.265\end{array}$ & $\begin{array}{l}58.583 \pm \\
13.007^{* * *}\end{array}$ & $\begin{array}{c}53.269 \pm \\
18.097\end{array}$ & $\begin{array}{c}53.101 \pm \\
22.778\end{array}$ & $\mathrm{P}<0.01$ & $\mathrm{P}<0.01$ \\
\hline & II & $\begin{array}{c}6.845 \pm \\
1.489^{\mathbf{b}}\end{array}$ & $\begin{array}{c}7.315 \pm \\
2.268^{b}\end{array}$ & $\begin{array}{c}10.894 \pm \\
4.492\end{array}$ & $\begin{array}{c}15.694 \pm \\
5.532\end{array}$ & $\begin{array}{c}40.447 \pm \\
8.673^{\mathrm{c}}\end{array}$ & $\begin{array}{c}56.437 \pm \\
22.304\end{array}$ & $\begin{array}{c}22.459 \pm \\
4.663^{\mathrm{c}}\end{array}$ & $\begin{array}{c}20.107 \pm \\
8.796^{\mathbf{b}}\end{array}$ & $\mathrm{P}<0.01$ & $\mathrm{P}<0.01$ \\
\hline \multirow{2}{*}{ Gills } & I & $\begin{array}{c}19.433 \pm \\
6.682\end{array}$ & $\begin{array}{c}25.994 \pm \\
10.768\end{array}$ & $\begin{array}{c}24.769 \pm \\
9.502\end{array}$ & $\begin{array}{c}26.311 \pm \\
10.356\end{array}$ & $\begin{array}{c}36.875 \pm \\
17.979\end{array}$ & $\begin{array}{c}19.184 \pm \\
9.693^{*}\end{array}$ & $\begin{array}{c}35.690 \pm \\
16.638\end{array}$ & $\begin{array}{c}39.702 \pm \\
17.927\end{array}$ & N.S. & N.S. \\
\hline & II & $\begin{array}{c}40.338 \pm \\
13.435^{c}\end{array}$ & $\begin{array}{c}27.848 \pm \\
12.303^{*}\end{array}$ & $\begin{array}{c}12.548 \pm \\
4.180^{\mathrm{b}}\end{array}$ & $\begin{array}{l}37.285 \pm \\
15.410^{* * *}\end{array}$ & $\begin{array}{c}84.728 \pm \\
29.739^{c}\end{array}$ & $\begin{array}{c}66.600 \pm \\
15.918^{c}\end{array}$ & $\begin{array}{c}33.375 \pm \\
14.710\end{array}$ & $\begin{array}{c}36.640 \pm \\
7.424\end{array}$ & $\mathrm{P}<0.01$ & $\mathrm{P}<0.01$ \\
\hline \multirow{2}{*}{ Skin } & I & $\begin{array}{c}9.116 \pm \\
4.241\end{array}$ & $\begin{array}{c}8.593 \pm \\
0.986\end{array}$ & $\begin{array}{c}11.170 \pm \\
4.893\end{array}$ & $\begin{array}{c}9.099 \pm \\
4.765\end{array}$ & $\begin{array}{c}18.229 \pm \\
5.160\end{array}$ & $\begin{array}{l}50.651 \pm \\
21.727^{* * *}\end{array}$ & $\begin{array}{c}38.950 \pm \\
16.438\end{array}$ & $\begin{array}{c}38.820 \pm \\
19.038\end{array}$ & $\mathrm{P}<0.01$ & $\mathrm{P}<0.01$ \\
\hline & II & $\begin{array}{l}5.275 \pm \\
1.147^{\mathrm{a}} \\
\end{array}$ & $\begin{array}{l}5.637 \pm \\
1.748^{c} \\
\end{array}$ & $\begin{array}{c}8.395 \pm \\
3.462 \\
\end{array}$ & $\begin{array}{c}12.094 \pm \\
4.263 \\
\end{array}$ & $\begin{array}{c}31.170 \pm \\
6.683^{\mathrm{c}} \\
\end{array}$ & $\begin{array}{c}43.492 \pm \\
17.188 \\
\end{array}$ & $\begin{array}{c}17.308 \pm \\
3.594^{\mathrm{c}} \\
\end{array}$ & $\begin{array}{c}15.496 \pm \\
6.779^{\mathbf{b}} \\
\end{array}$ & $\mathrm{P}<0.01$ & $\mathrm{P}<0.01$ \\
\hline \multirow{2}{*}{ Muscles } & I & $\begin{array}{c}5.775 \pm \\
2.671\end{array}$ & $\begin{array}{c}5.049 \pm \\
2.617\end{array}$ & $\begin{array}{c}2.846 \pm \\
0.601\end{array}$ & $\begin{array}{c}3.797 \pm \\
1.754\end{array}$ & $\begin{array}{c}3.608 \pm \\
1.305\end{array}$ & $\begin{array}{c}3.983 \pm \\
1.624\end{array}$ & $\begin{array}{c}1.991 \pm \\
0.840\end{array}$ & $\begin{array}{c}2.280 \pm \\
0.527\end{array}$ & $\mathrm{P}<0.05$ & N.S. \\
\hline & II & $\begin{array}{c}2.295 \pm \\
1.122^{\mathrm{b}}\end{array}$ & $\begin{array}{l}2.147 \pm \\
1.301^{b}\end{array}$ & $\begin{array}{c}3.452 \pm \\
1.048\end{array}$ & $\begin{array}{c}2.961 \pm \\
1.124\end{array}$ & $\begin{array}{c}5.540 \pm \\
1.135^{\mathbf{b}}\end{array}$ & $\begin{array}{l}3.097 \pm \\
1.278^{* * *}\end{array}$ & $\begin{array}{c}1.545 \pm \\
0.720\end{array}$ & $\begin{array}{c}1.161 \pm \\
0.284^{c}\end{array}$ & $\mathrm{P}<0.01$ & N.S. \\
\hline
\end{tabular}

See footnote table 3

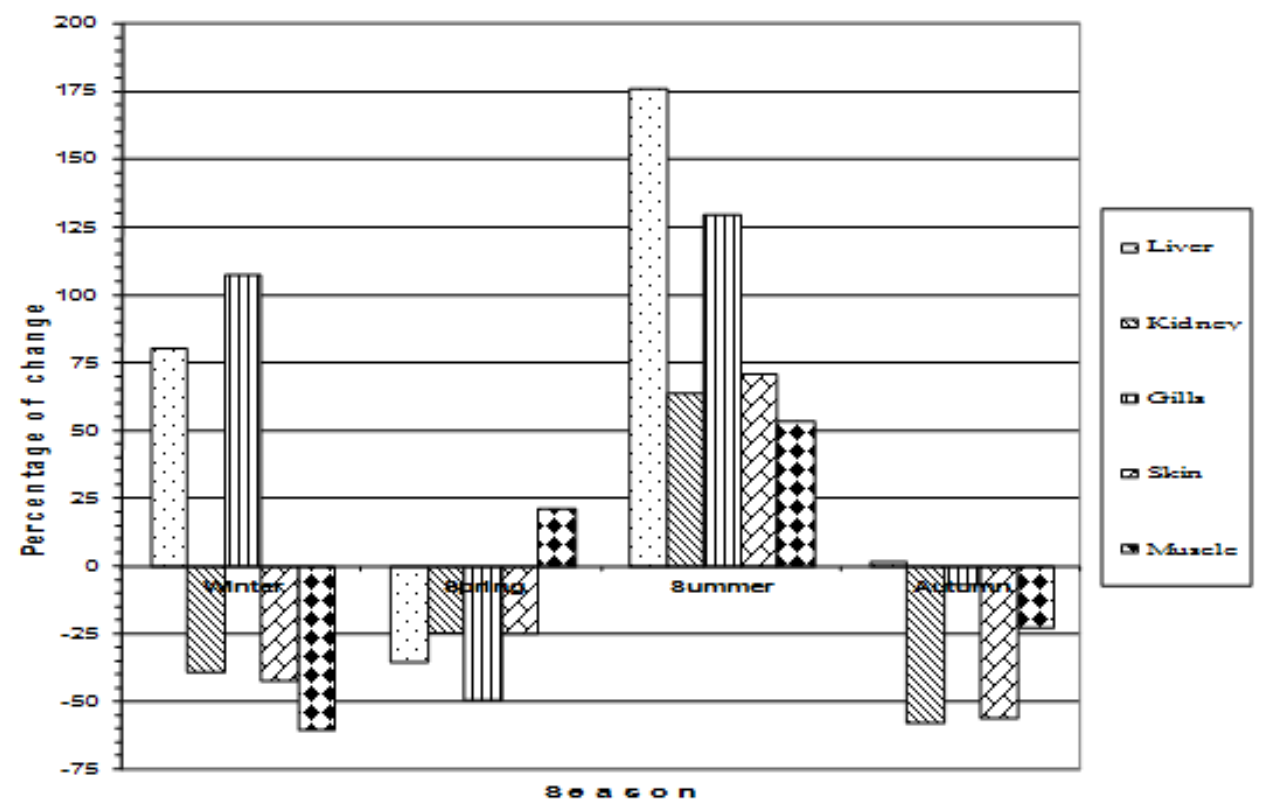

Fig. 8: The percentage of change in the manganise concentrations in the liver, kidney, gills skin and muscles of male Clarias gariepinus from Barrage (site II) relative to Al-Aiat (site I) during the four seasons. 


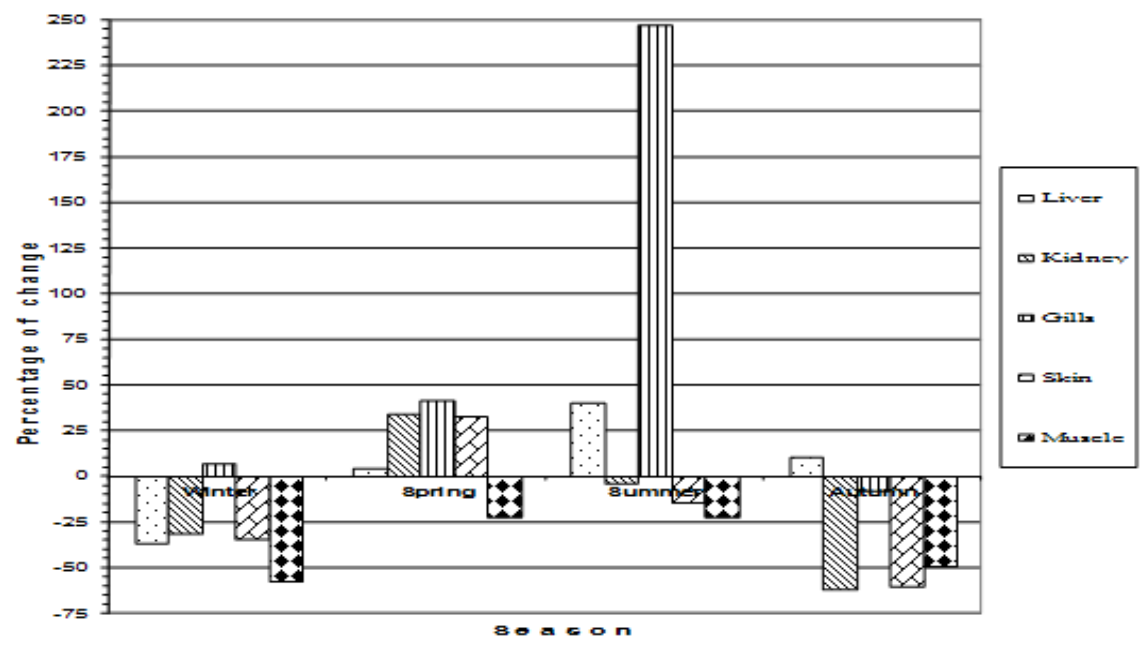

Fig. 9: The percentage of change in the manganise concentrations in the liver, kidney, gills skin and muscles of female Clarias gariepinus from Barrage (site II) relative to Al-Aiat (site I) during the four seasons.

\section{2) Effect of sex:}

\section{Iron (Table 3):}

At winter, only liver Fe in female fish from Al-Aiat region had a significant $(\mathrm{P}<$ 0.05) higher value than male ones. At spring, females from Barrage area had significant $(\mathrm{P}<0.05)$ higher values of the kidney and skin Fe than males. At summer, kidney Fe was the only value that significantly $(\mathrm{P}<0.05)$ higher in female fish from Al-Aiat area than male ones. At autumn, significant lower values of Fe in both liver ( $\mathrm{P}$ $<0.05)$ and muscles $(\mathrm{P}<0.01)$ and significant higher value in the kidney $(\mathrm{P}<0.01)$ of females from Al-Aiat area were demonstrated compared to the males. Fish collected from Barrage area showed significant higher values of Fe in both liver $(\mathrm{P}<0.05)$ and muscles $(\mathrm{P}<0.01)$ and significant lower values in the gills $(\mathrm{P}<0.05)$ than males.

\section{Copper (Table 4):}

Female fish collected from Al-Aiat region demonstrated significant $\mathrm{Cu}$ values compared to males as follows: higher values in the liver at winter $(\mathrm{P}<0.01)$ and spring $(\mathrm{P}<0.05)$; higher value $(\mathrm{P}<0.01)$ in the kidney at summer; lower and higher values $(\mathrm{P}<0.01)$ in the gills at spring and autumn respectively; lower value $(\mathrm{P}<0.01)$ in the skin at winter and higher values $(\mathrm{P}<0.05)$ at both spring and autumn; and higher value $(\mathrm{P}<0.05)$ in the muscles at spring. The only significant $(\mathrm{P}<0.05)$ higher value in female fish from Barrage area was detected in the liver at autumn than males.

\section{Zinc (Table 5):}

At summer, female fish showed significant $(\mathrm{P}<0.01)$ higher values of $\mathrm{Zn}$ in the kidney (from Al-Aiat) and skin (from both areas) and lower value in the muscles (from Al-Aiat) than the respective males. At autumn, the only significant $(\mathrm{P}<0.05)$ value was detected in the skin of female fish from Al-Aiat area that showed higher value than male.

\section{Manganese (Table 6):}

Female fish from Al-Aiat area demonstrated the following significant values of Mn compared to the males: higher values in the liver at both winter $(\mathrm{P}<0.001)$ and summer $(\mathrm{P}<0.01)$; higher values $(\mathrm{P}<0.001)$ in both kidney and skin at summer; and lower value $(\mathrm{P}<0.05)$ in the gills at summer. Female fish from Barrage area represent the following: higher values $(\mathrm{P}<0.001)$ in both liver and gills at spring; lower value $(\mathrm{P}<0.05)$ in the gills at winter; and lower value $(\mathrm{P}<0.001)$ in the muscles at summer. 


\section{3) Effect of season: Iron (Table 3):}

Almost the maximum and minimum values of the iron in the five organs were recorded at winter and spring respectively. The maximum values were detected as follows: for liver in both sexes from Al-Aiat and males from Barrage; for muscles in both sexes from Barrage and females from Al-Aiat; and for kidney in males from both locations. Another peak of the liver Fe was observed at autumn in male fish from AlAiat. The minimum values were observed as follows: for liver and muscles in both sexes from both areas; for kidney in both sexes from Al-Aiat and males from Barrage; and for skin in males from both locations. Another lower value of the muscles Fe was shown in male fish from Barrage at autumn. The maximum and minimum values of $\mathrm{Fe}$ in the gills of both sexes from Barrage were detected at spring and autumn respectively. The maximum values of $\mathrm{Fe}$ in the skin were found at summer for both sexes from both locations. Females from both areas recorded maximum values of Fe in the kidney at autumn. The minimum values of Fe in the gills of both sexes from AlAiat were found at winter. The other maximum and minimum values were illustrated in table 3. ANOVA analysis demonstrated that season significantly affects on the Fe concentrations as follows: in the liver of both sexes from the two localities; in the kidney of both sexes from Al-Aiat; and in the skin and muscles of males from Al-Aiat and Barrage areas respectively.

\section{Copper (Table 4):}

Copper concentrations in the kidney of all fish groups showed the maximum and minimum values at autumn and spring respectively. Also the maximum values of $\mathrm{Cu}$ in the muscles of the four groups were recorded at autumn but the minimum values were at winter except males from Al-Aiat at spring. Both sexes from Barrage showed maximum and minimum values of $\mathrm{Cu}$ in the liver at summer and spring respectively, but another lower value was observed at winter for females. The $\mathrm{Cu}$ values in the gills of both sexes from Barrage were highest and lowest at winter and summer respectively. Both sexes from Al-Aiat and females from Barrage recorded the maximum values of $\mathrm{Cu}$ in the skin at summer, while both sexes from Barrage and females from $\mathrm{Al}$-Aiat recorded the minimum values at winter. The other maximum and minimum values were demonstrated in table 4. Copper contents in the kidney, skin and muscles of both sexes from both locations were significantly affected by season. Also ANOVA showed that gill's $\mathrm{Cu}$ in both sexes from Barrage and liver's $\mathrm{Cu}$ in females from Al-Aiat were significantly $(\mathrm{P}<0.05)$ affected by season.

\section{Zinc (Table 5):}

The maximum concentrations of $\mathrm{Zn}$ in the liver of both sexes from Al-Aiat and Barrage were detected at winter and summer respectively, while the minimum values for all were recorded at spring except the female fish from barrage at winter. For $\mathrm{Zn}$ in the kidney, the minimum values for all groups were observed at spring, while the maximum values were demonstrated at winter except the female fish from barrage at summer. For $\mathrm{Zn}$ in the gills, higher values for both sexes from Al-Aiat were detected at summer and the lower values at autumn and winter for males and females respectively. Fish from Barrage did not show a typical pattern for both sexes. For Zn in the skin, the maximum values in both sexes from Al-Aiat and Barrage were shown at autumn and summer respectively, while the minimum values in both sexes from Barrage were detected at winter. For $\mathrm{Zn}$ in the muscles, the maximum values in males and females from both areas were recorded at summer and spring respectively, while the minimum values for both sexes from Barrage were detected at winter. The other values were illustrated in table 5 . ANOVA demonstrated that $\mathrm{Zn}$ concentration in the 
liver and skin (Barrage); kidney (Al-Aiat) of both sexes; kidney (Barrage) of males; and skin (Al-Aiat) of females were affected by season.

\section{Manganese (Table 6):}

An obvious similarity was observed between the concentrations of $\mathrm{Mn}$ in both kidney and skin of both sexes from both areas. The minimum values were recorded at winter for all groups, while the maximum values were detected at summer except males from Al-Aiat at autumn. Another similarity was illustrated between $\mathrm{Mn}$ contents in both liver and gills. The minimum values were at winter for males (AlAiat) and females (Barrage); at spring for males (Barrage); and at summer for females (Al-Aiat). The maximum values of liver's Mn were at autumn for all groups except males from Barrage at summer, contrary values of gill's Mn were at summer except females from Al-Aiat at autumn. The Mn contents in the muscles demonstrated maximum values at winter and summer for both sexes from Al-Aiat and Barrage respectively, while the minimum values were at autumn for all groups. Manganese contents in the kidney and skin (both sexes from both areas); in the liver and gills (both sexes from Barrage); in the liver (males from Al-Aiat); and in the muscles (males from both areas) were affected by season as analyzed by ANOVA test.

\section{4) Ranking of heavy metals in organs:}

Tables 7 and 8 illustrate the distribution of the four elements in the five organs of both sexes in both localities. Iron and copper are similar in their distributions in both sexes, in which liver is the main site of accumulation followed by the kidney then gills. Muscles are the last organ that accumulates Fe in both sexes, while skin is the last organ that accumulate $\mathrm{Cu}$ in males and equal to the muscles in females. Kidney and gills in both sexes are the main organs that accumulate $\mathrm{Zn}$ and $\mathrm{Mn}$ respectively. For $\mathrm{Zn}$, liver is the second organ followed by the gills and lastly the muscles. Skin is equal to the gills for $\mathrm{Zn}$ accumulation in case of females. For $\mathrm{Mn}$, kidney is the second organ followed by the skin and lastly the liver (males) or muscles (females).

Table 7: Ranking of iron, copper, zinc and manganese distribution in different organs of male Clarias gariepinus collected from Al-Aiat and Barrage areas allover the year.

\begin{tabular}{|c|c|c|c|c|c|}
\hline Metal & Liver & Kidney & Gills & Skin & Muscle \\
\hline $\mathrm{Fe}$ & 1 & 2 & 3 & 4 & 5 \\
\hline $\mathrm{Cu}$ & 1 & 2 & 3 & 5 & 4 \\
\hline $\mathrm{Zn}$ & 2 & 1 & 3 & 4 & 5 \\
\hline $\mathrm{Mn}$ & 5 & 2 & 1 & 3 & 4 \\
\hline
\end{tabular}

Table 8: Ranking of iron, copper, zinc and manganese distribution in different organs of female Clarias gariepinus collected from Al-Aiat and Barrage areas allover the year.

\begin{tabular}{|c|c|c|c|c|c|}
\hline Metal & Liver & Kidney & Gills & Skin & Muscle \\
\hline $\mathrm{Fe}$ & 1 & 2 & 3 & 4 & 5 \\
\hline $\mathrm{Cu}$ & 1 & 2 & 3 & 4 & 4 \\
\hline $\mathrm{Zn}$ & 2 & 1 & 3 & 3 & 4 \\
\hline $\mathrm{Mn}$ & 4 & 2 & 1 & 3 & 5 \\
\hline
\end{tabular}

\section{DISCUSSION}

Allover the year, the present water Fe levels in both Al-Aiat and Barrage areas (table 2) are mush lower than that recorded by Issa et al. (1997) in both Helwan (8.84$10.54 \mathrm{mg} \mathrm{l}^{-1}$ ) and Barrage (0.364 $\mathrm{mg} \mathrm{l}^{-1}$ ). Khalaf-Allah (1998) reported that the mean value of Fe concentration in water samples around Helwan exceeded the permissible limits set by WHO (1984). Contrary to Issa et al. (1997) who mentioned that the Fe 
content in the Nile water is slightly affected by water temperature, the present data did not reveal any seasonal variation (table 2). El-Nady (1981) and Issa et al. (1997) noted that there is a negative correlation between dissolved heavy metals and dissolved oxygen. It is known that ferrous (which is believed to be toxic) and ferric salts are usually soluble in areas poor in dissolved oxygen (Hem, 1989). Wetzel (1983) and Hem (1989) stated that the occurrence of Fe in water strongly influenced by the presence of organic wastes and plant debris in soil. On the other hand, Rizkalla and Abo-Donia (1996a) found that phytoplankton evidently was one of the factors causing the removal of the Fe from water.

Water copper levels in Barrage area at both winter and autumn are significantly lower than that in Al-Aiat area (table 2). The Cu levels in both areas are much lower than that recorded by Issa et al. (1997) in Helwan and Barrage areas (4.63-30.57 $\mathrm{mg} \mathrm{l}^{-1}$ ). Working on the River Nile water around Helwan, Khalaf-Allah (1998) reported that the mean value of $\mathrm{Cu}$ concentrations were within the guide line values of WHO (1984). This lower value was confirmed by Daoud et al. (1999) on the $\mathrm{Cu}$ and $\mathrm{Zn}$ concentrations in water from Helwan. The calculated global averages for river water were presented by Liebman (1960) to be $7 \mu \mathrm{g} / \mathrm{l}$. Alabaster and Lloyd (1980) recorded that the natural concentrations of $\mathrm{Cu}$ in water are at $<5 \mu \mathrm{g} / \mathrm{l}$, but according to the Department of Water Affairs and Forestry $\left(1993_{\mathrm{b}}\right.$ ) levels of $12 \mathrm{mg} / \mathrm{l}$ have been recorded in South Africa. The aqueous $\mathrm{Cu}$ exposure studies on O. niloticus confirm that $\mathrm{Cu}$ can be acutely toxic at around 1-5 $\mu \mathrm{g} \mathrm{l^{-1 }}$ total $\mathrm{Cu}$ (Coğun and Kargin, 2004). According to Stiff (1971), the soluble forms of $\mathrm{Cu}$ are the only forms available to fish. The toxicity of $\mathrm{Cu}$ appears to be related to the soluble form, with $\mathrm{Cu}^{2+}$ and, to some extent, the $\mathrm{Cu}$ hydroxyl ion being the toxic forms. The free ion rarely occurs in aquatic environments as the soluble form of $\mathrm{Cu}$ in river waters and consists mainly of complexed, non-toxic forms. Lloyd (1992) found that the main environmental factor that affects copper toxicity in water is calcium concentration. This is as a result of the competition that occurs between these two ions for binding sites in the tissue of the gills and other organs. Copper in the alkaline conditions precipitates as the carbonate and is non toxic (Dallas and Day, 1993). Humic acids, hardness, temperature, $\mathrm{pH}$, dissolved oxygen and suspended solids can have a significant altering effect on the bioavailibility of Cu to fish (Stiff, 1971; Moore and Ramamoorthy, 1984). The results of Issa et al. (1997) indicated that $\mathrm{Cu}$ is negatively correlated with water transparency and dissolved oxygen as a result of the formation of insoluble $\mathrm{CuO}$ and is positively correlated with total dissolved substances.

The records of Issa et al. (1997) on Zn distribution in River Nile showed two peaks in the surface water at Helwan (375.7 $\mathrm{mg} \mathrm{l}^{-1}$ ) during summer and $299.77 \mathrm{mg} \mathrm{l}^{-1}$ at Barrage during spring and the minimum value $\left(20.76 \mathrm{mg} \mathrm{l}^{-1}\right)$ is shown at Helwan during spring season. These data are much higher than that recorded in the present study (table 2). The calculated global averages for river water were presented by Liebman (1960) to be $20 \mu \mathrm{g} / \mathrm{l}$. Issa et al. (1997) related the highly significant positive correlation between $\mathrm{Zn}$ and suspended matter to adsorption of zinc with anhydrous $\mathrm{Fe}_{2} \mathrm{O}_{3}$ and $\mathrm{Al}_{2} \mathrm{O}_{3}$ which acts as scavenger for zinc. On the other hand, the highly significant negative correlation between $\mathrm{Zn}$ and dissolved oxygen is attributed to the increase of the precipitation of zinc as $\mathrm{Zn}(\mathrm{OH})_{2}$ at high $\mathrm{pH}$ in the presence of oxygen. Hassel et al. (1980) found that concentrations of $\mathrm{Zn}$ was low in stream water samples obtained at sites adjacent to highways and stream sediments appeared to serve as the strong reservoir and primary source for bioaccumulation of heavy metals.

No significant difference in the concentrations of manganese in the water of River Nile between Al-Aiat and Barrage areas were observed except at summer (table 
2). The lowest concentration of Mn during summer in Barrage area may be attributed to uptake of Mn by phytoplankton in which the dead phytoplankton is settled to the sediment leaving the water poor in the trace metal (Issa et al., 1997). They reported that $\mathrm{Mn}$ shows the highest concentration at Helwan (552.76 $\mathrm{mg} \mathrm{l}^{-1}$ ) in winter season and the lower of (17.14 $\mathrm{mg} \mathrm{l}^{-1}$ ) during summer. Barlas (1999) found that concentration of water $\mathrm{Cu}$ and $\mathrm{Mn}$ differed by seasons. Rizkalla and Abo-Donia (1996a) reported that $\mathrm{Fe}$ and $\mathrm{Mn}$ in River Nile water of Upper Egypt indicated significant variations with region. Issa et al. (1997) said that Mn is: slightly correlated with temperature; negatively correlated with dissolved oxygen (due to oxidation of Mn and uptake of it by the phytoplankton); and highly significant positive correlated with total suspended substances (due to the adsorption of Mn on the surface of suspended sediments). The toxicity of manganese is not dependent on the total manganese concentration, but the concentration of oxidized manganese which is available (Clement Associates, 1985). Higher concentrations of $\mathrm{Ca}^{2+}$ ions will compete with $\mathrm{Mn}$ ions for absorption through the gill surface thus decreasing the bioavailability of manganese to fish (Seymore et al., 1995).

Rizkalla and Abo Donia (1996, $\mathrm{a}$ ) and Daoud et al. (1999) pointed to the idea that the concentrations of heavy metals in the aquatic environment can be correlated to their concentrations in the bodies of animals surviving in such environment. The exchange processes between the metallic trace elements of water and the fish that live therein is dependent upon factors influenced by the environment and upon biological factors (Harms, 1975). Fast et al. (1990) indicated in their experiment that metal uptake from the water was not of significant concern and the principal source of heavy metals in salmon was from diet. Rizkalla and Abo Donia (1996a) computed that deboned flesh concentrations of $\mathrm{Fe}$ and $\mathrm{Mn}$ in Oreochromis niloticus were bioaccumulated from water, and Zn from sediment. Gaber (2008) revealed significant positive correlations between heavy metals concentrations in tilapia flesh and water.

Significant variation among individuals was observed for all bioaccumulated metals tested and in all the organs sampled. Also, concentration differences were observed for the individual metals in the same organs. The differences in tissue metal concentrations of fish from a specific locality were cited by Du Preez and Steyn (1992). The present results revealed that liver is the main organ of iron accumulation in both sexes of $C$. gariepinus and the maximum and minimum concentrations occurred in winter and spring respectively (table 3). Avenant-Oldewage and Marx (2000) concluded the same observation on the same fish species. The liver has a vast vascular network where blood passes through. Iron released from the breakdown of haemoglobin, as well as excess Fe found in the body, is stored and detoxified in the liver (Buckley et al., 1982). Kidney is the $2^{\text {nd }}$ organ that accumulates Fe (table 3). Rizkalla et al. (2003) illustrated that $\square$ kidney, spleen and liver are the main sites of Fe deposition. Gills are the $3^{\text {rd }}$ organ that accumulates $\mathrm{Fe}$, and the maximum concentrations were recorded at spring while the minimum concentrations were at winter and autumn for fish collected from Al-Aiat and Barrage respectively (table 3). Avenant-Oldewage and Marx (2000) said that the high concentrations of Fe found in the sediment could have resulted in the high Fe levels in the gills during the process of bottom feeding of C. gariepinus. Laurent and Dunel (1980) mentioned that with an immense vascular network in close proximity to the gill tissue, the gills will be in continual contact with the blood-borne Fe. Finally, the minimal concentrations of Fe were detected in muscles (table 3). Working on C. gariepinus, Avenant-Oldewage and Marx (2000) reported that the low storage of iron in muscle would be accompanied by low iron concentrations in the skin and no significant difference were found between 
them. On the other hand, Adeyeye et al. (1996) and Olaifa et al. (2004 $)$ observed that $\mathrm{Fe}$ is the dominant metal in the muscle of $C$. gariepinus. Some scattered significant differences in the concentrations of Fe in different organs (especially skin) between Al-Aiat and Barrage areas were detected (table 3). Rizkalla and Abo Donia (1996a) referred that after $\mathrm{Zn}$, Fe is the most common metallic trace element in deboned flesh of Oreochromis niloticus and their data supported the impression that metal-specific differences in the concentration levels existed between different localities. The significant seasonal variations in the Fe concentrations were obviously detected in the liver of both sexes from both localities (table 3). Avenant-Oldewage and Marx (2000) showed seasonal changes in the concentration of $\mathrm{Fe}$ in the liver of $C$. gariepinus as well as in the skin and muscle. As previously mentioned, increasing water temperatures enhance the oxidation of the soluble $\mathrm{Fe}^{2+}$ to the insoluble $\mathrm{Fe}^{3+}$ iron states. The abiotic factors mentioned above are also at a maximum level during winter (Department of Water Affairs and Forestry, 1993a).

As iron, the major site for the accumulation of copper is the liver (table 4). Hogstrand et al. (1991) and Carvalho et al. (2004 ${ }_{\mathrm{a}}$ ) demonstrated high copper accumulation in the livers of Perca fluviatilis and Prochilodus scrofa with concomitant expression of metallothioneins (MT) in the liver. However, it reinforces the view that the liver in fish plays a protective role against chronic heavy metal exposures by producing MT (Carpene and Vašák, 1989), acting as a storage site, and being a vital organ in the detoxification mechanisms of copper (Buckley et al., 1982 and Mayer et al., 2003). Kidney is the $2^{\text {nd }}$ organ that accumulates $\mathrm{Cu}$ and the maximum and minimum concentrations were at autumn and spring respectively (table 4). The highest copper concentration in the kidney of Clarias gariepinus (Farombi et al., 2007) may be due to the fact that fish kidney contains a cystine rich copper binding protein which is thought to have either a detoxifying or storage function as reported by Luckey and Venugopal (1977). Gills are the $3^{\text {rd }}$ organ that accumulates $\mathrm{Cu}$ (table 4). Clarias do not store significant higher levels of $\mathrm{Cu}$ in their gills, but Tilapia species showed significant higher concentrations of this metal in their gills (El-Shehawi et al., 2007). Shaw and Handy (2006) showed that Nile Tilapia fish accumulate excess $\mathrm{Cu}$ in the liver and gills. DeOliveira et al. (2004) reported that $\mathrm{Cu}^{2+}$ accumulated in tambaqui (Colossoma macropomum), in decreasing order, gills > liver. Several authors (Gatlin et al., 1989 and Miller et al., 1993) have shown that increased concentrations in the liver and gills were due to increased $\mathrm{Cu}$ in the diet. The gill tissue of the fish tends to concentrate $\mathrm{Cu}$ from the water either due to the gills possesses an extensive vascular network as previously mentioned by Laurent and Dunel (1980) or due to the short diffusion distance from the water to the blood, as well as the large surface area exposed to the water (Stagg and Shuttleworth, 1982). It has been concluded from the results of many studies that the accumulation of $\mathrm{Cu}$ in the gill is possibly due to its binding to haemopoeitic tissue, mucous and metallothioneins implicated with excretion and detoxification (Avenant-Oldewage and Marx, 2000). The lowest concentrations of $\mathrm{Cu}$ were recorded in the muscles with the maximum and minimum values at autumn and winter respectively (table 4). Higher $\mathrm{Cu}$ values were estimated by Seddek et al. (1996) in C. lazera muscles (5.55 ppm), but lower values $0.03 \pm 0.004 \mathrm{ppm}$ were recorded by Abd El-Khalek et al. (2002). Abd El-Nasser et al. (1996) revealed an elevation of $\mathrm{Cu}$ in Oreochromis niloticus muscle in comparison to C. lazera. These findings contradict that of Hassan and Youssef (1985) who reported that $\mathrm{Cu}$ concentration in muscles of C. lazera was higher than that of Tilapia, while little difference was found by Ghazaly et al. (1992) and Rizkalla et al. (2003) between the metal content of the two species. According to 
Moore and Ramamoorthy (1984), fish muscle normally contains low concentrations of $\mathrm{Cu}$ and, even at high levels of $\mathrm{Cu}$ exposure; muscle does not often reflect increases in the external environment. Contrary to $\mathrm{Fe}, \mathrm{Cu}$ concentrations in different organs recorded large numbers of significant values between Al-Aiat and Barrage areas, while significant sex variation was fewer (table 4). Ghazaly et al. (1992) and Rizkalla et al. (2003) found that $\mathrm{Cu}$ level in the muscles of C. lazera and O. niloticus was affected by the locality and $\mathrm{Cu}$ concentration in gills from three different localities had a small range of variation. Significant difference in the concentrations of $\mathrm{Cu}$ in the muscles of both sexes of Sardinella aurita was reported by Shakweer and Abbas (1996). An obvious significant seasonal effect was demonstrated in the concentrations of $\mathrm{Cu}$ in the kidney, skin and muscles (table 4). The higher the temperature, the more ionized copper forms are produced resulting in greater toxicity to the fish (Prosi, 1979). The seasonal pattern observed by Avenant-Oldewage and Marx (2000) showed that in winter bioaccumulation was at a minimum, while generally in summer bioaccumulation rates were at a maximum. The effect of temperature seems to be evident in the MT concentration (Carvalho et al., 2004 ${ }_{\mathrm{a}, \mathrm{b}}$ ).

The main storing site of zinc in both sexes of $C$. gariepinus collected from AlAiat and Barrage areas is the kidney followed by the liver (tables 5, $7 \& 8$ ). The high accumulation of $\mathrm{Zn}$ in the kidney could be based on specific metabolism process and co-enzyme catalyzed reactions involving $\mathrm{Zn}$ taking place in the kidney (Jaffar and Pervaiz, 1989). Zn also acts as a catalyst in metal biomolecules bound to amino acid side chains containing N, O and/or sulphur legends (Kendrick et al., 1992) to form tetrahedral zinc metalloproteins and metalloenzymes in kidney tissues (Ashraf, 2005). High concentration of $\mathrm{Zn}$ was reported in the liver of seven fish species from the coastal waters of Uruguay (Viana et al., 2005). Also, Annune and Iyaniwura (1993) reported the liver of Oreochromis niloticus and Clarias gariepinus to have accumulated Zn more than other tissues. Moreover, Farombi et al. (2007) observed high accumulation of $\mathrm{Zn}$ in the liver and gills of $C$. gariepinus. The high level of $\mathrm{Zn}$ in the liver may possibly reflect the multifunctional role of the liver in the detoxification (through metallothioneins binding) and storage processes (Carpene et al., 1990). Gills are the $3^{\text {rd }}$ organ that accumulates $\mathrm{Zn}$, but in the female fish skin takes the same level (tables 7 \& 8). Annune and Iyaniwura (1993) and Gbem et al. (2001) studied the bioaccumulation of trace metals in the gills of several fish species including $C$. gariepinus. The highest accumulation of $\mathrm{Zn}$ in the gills may be due to the facts that gills are in direct contact with the contaminated water; have the thinnest epithelium of all the organs; metals can penetrate through the thin epithelial cells (active process) and serves as the respiratory organ through which metal ions are absorbed (Bebianno et al., 2004). The present results agreed with Badsha and Goldspink (1982); Annune and Iyaniwura (1993); Gbem et al. (2001) and Birungi et al. (2007) that the lowest Zn loads were found in the muscles. The $\mathrm{Zn}$ concentration in $C$. lazera dry muscle recorded by Abd El-Khalek et al. (2002) was $0.27 \pm 0.03$ ppm. This might be attributed to the growth factor as growth may dilute toxicant concentration if growth is faster than accumulation (Badsha and Goldspink, 1982). Jeng and Sun (1981) fed high levels of Zn sulfate to Cyprinus carpio and found $\mathrm{Zn}$ levels to be higher in the skeletal tissues, and that muscle metal levels were not increased till skeletal tissues are saturated. No clear sex differences were recorded (table 5). Shakweer and Abbas (1996) found that the differences between $\mathrm{Zn}$ concentrations in the muscles of the males and females of Oreochromis niloticus and Sardinella aurita were not significant while its concentration in the liver of the females of Sardinella aurita differed significantly from the corresponding concentrations in the males. 
The highest values of manganese were recorded in the gills of both sexes of $C$. gariepinus from both sites studied (table 6). This result was confirmed by the results of Abdel-Baky (2001) on the same fish species. The mean Mn concentration in gills of C. gariepinus was $0.014 \mathrm{ppm}$ in the dry season and $0.063 \mathrm{ppm}$ in the wet season (Olaifa et al., 2004b). Also, working on 230 fish from the Rockall Trough (NorthEastern Atlantic), Vas et al. (1993) found that Mn accumulated mainly in gill tissues. The high accumulation of $\mathrm{Mn}$ in gill tissues may be attributed to the complex formation between metal ion and the protein structure in gills which contain nitrogen, oxygen and/or sulfur as reported by Cotton and Wilkinson (1980). Liver and muscles showed the least organs that accumulate Mn (tables 6 - 8). Olaifa et al. (2004b) recorded higher concentrations of manganese in muscle tissue of C. gariepinus (2.17 $\mathrm{ppm}$ in the dry season and $0.315 \mathrm{ppm}$ in wet season). Among the metals, Mn was lowest in the muscle of mullet (Liza $a b u$ ) and catfish (Silurus triostegus) from the Atatürk Dam Lake, Turkey (Karadede et al., 2004). Significant location and seasonal variations were recorded in the Mn concentration in different organs with maximum and minimum values at summer and winter respectively (table 6). No significant variation was recorded by Olaifa et al. $\left(2004_{b}\right)$ between stations and seasons in $\mathrm{Mn}$ concentrations in muscles of $C$. gariepinus. Also but to a least extend, significant sex variation was detected (table 6). Shakweer and Abbas (1996) observed that the flesh of the males of Sardinella aurita contained significantly higher concentrations of $\mathrm{Mn}$ than that in the females.

Wepener et al. (2001) said that gill tissue is the initial site of accumulation of water-borne metals. Although the fish were exposed to a metal mixture, the interactions between metals and the external gill surface, as well as the subsequent uptake rate, were associated with the particular chemical properties of individual metals. The tendency of the individual metals in the metal mixture to bind to the external gill surface via ionic bonds, and to gill cytosolic compounds via covalent bonds, was $\mathrm{Cu}>\mathrm{Fe}>\mathrm{Zn}$. Roméo et al. (2005) found that gills presented the highest $\mathrm{Cu}$ and $\mathrm{Zn}$ concentrations compared to the visceral mass or the remaining tissues. The gills and the liver are more often recommended as environmental indicator organs of water pollution than any other fish organs (Al-Yousuf et al., 2000; Arain et al., 2008). These two tissues have the highest metal burdens and represent the bioavailability of metal concentrations in the environment. The gills are directly in contact with water. Thus, the concentrations of metals in the gills reflect the concentrations of metals in the waters, whereas the concentrations in liver represent storage of metals (Rao and Padmaja, 2000). In general, Birungi et al. (2007) concluded that the order of accumulation of metals in tissue of Oreochromis niloticus was $\mathrm{Cu}>\mathrm{Zn}>\mathrm{Mn}$ and gills $>$ liver $>$ muscle.

The effect of locality on metal concentrations in teleosts was highly significant as recorded by Walker (1982). Niazzy et al. (1995) revealed significant variations in the concentrations of $\mathrm{Cu}, \mathrm{Fe}$ and $\mathrm{Zn}$ in both livers and muscles of Clarias lazera trapped from different localities in Egypt and the data collected by Rizkalla and Abo Donia (1996a) supported the impression that significant metal-specific differences in the concentration levels of $\mathrm{Cu}, \mathrm{Fe}, \mathrm{Mn}$ and $\mathrm{Zn}$ in muscle tilapia fish existed between the different localities from the upper section of the River Nile. Also, intra-location differences were detected in C. gariepinus for $\mathrm{Zn}$ and Mn concentrations in the gills; $\mathrm{Cu}$ and $\mathrm{Zn}$ concentrations in the muscles (Olaifa et al., 2004b) and $\mathrm{Cu}, \mathrm{Zn}$ and $\mathrm{Mn}$ in the muscles, liver and kidney (Abdel-Baky, 2001). Chernoff and Dooley (1979) reported significant differences between males and females with respect to wholebody $\mathrm{Zn}$ and $\mathrm{Cu}$ concentrations, but no sex difference for Mn. Also, Rizkalla et al. 
(2003) declared significant variation between male and female fishes from Mariut Lake and $\mathrm{Mn}$ is the least heavy metal shows sexual difference. Saleh et al. (1988) mentioned that $\mathrm{Cu}$ and $\mathrm{Zn}$ concentrations in Tilapia zillii from Wadi El-raiyan Lakes did not change significantly with the season of collection, while Oreochromis niloticus tissues showed seasonal variations in the concentrations of $\mathrm{Fe}, \mathrm{Zn}$ and $\mathrm{Cu}$. Also, Olaifa et al. (2004b) reported significant seasonal differences in the concentrations of $\mathrm{Cu}$ and $\mathrm{Zn}$ in the gills of $C$. gariepinus and $\mathrm{Cu}$ and $\mathrm{Fe}$ in the muscles. The increased accumulation of heavy metals in fish tissues during winter and autumn might be due to the intensive feeding on phytoplankton and other organisms during these seasons (Saleh et al., 1988; Al-Nagaaway et al., 2009).

Working on C. gariepinus, metal accumulation was in the order of: $\mathrm{Zn}>\mathrm{Cu}>$ Mn (Abdel-Baky, 2001 and Farombi et al., 2007) or $\mathrm{Cu}>\mathrm{Zn}$ (Gbem et al., 2001). These orders of accumulation may be due to the permeability of C. gariepinus to these metals. There could be competition for protein-binding sites both at the mucosal cell level and in tissues. Possible mechanisms of metal-metal interaction include interchange of metal bound proteins; induction of metal-binding protein; and formation of compound complexes among metals and metalloids (Gbem et al., 2001).

All five tissue types tested in the present work reflected differences when compared to each other, indicating a high degree of variability between these tissue types. Ranking of the different metals studied in the five organs (tables 7 \& 8) demonstrated that Fe was detected in all tissues sampled and C. gariepinus is able to bioaccumulate high levels of Fe. Previous literature existed great variations in the levels of Fe concentrations in the different tissues either liver $>$ gills $>$ muscle $>$ skin (Avenant-Oldewage and Marx, 2000) or kidney $>$ liver $>$ gills $>$ skin $>$ muscles (Rizkalla et al, 2003). For $\mathrm{Cu}$, differences in the $\mathrm{Cu}$ concentrations in the different organs of $C$. gariepinus were existed between the present study and other workers. The concentration in tissues was found to be in the following sequence: liver $>$ gills $>$ muscle $>$ skin (Avenant-Oldewage and Marx, 2000); liver $>$ gills $>$ skin $>$ muscle (Gbem et al., 2001); liver > kidney > gills > muscles (Abdel-Baky, 2001) or kidney > liver $>$ gills (Farombi et al., 2007). The livers of Oreochromis niloticus and Sardinella aurita (Shakweer and Abbas, 1996) and some fish species from Lake Mariut and Nozha Hydrodrome (Shakweer and Abbas, 1997) accumulate high concentrations of $\mathrm{Cu}$ if compared by its concentrations in the flesh or gonads. In the same trend, Rizkalla et al. (2003) reported that the highest and lowest concentrations of $\mathrm{Cu}$ are shown respectively in the liver and muscles of Oreochromis niloticus; C. gariepinus and Bagrus bayad. Also Buckley et al. (1982); Du Preez and Steyn (1992); and Vas et al. (1993) indicated that, in fish, Cu appeared to selectively accumulate in liver tissue and muscle tissue was a poor indicator of increased metal availability. In contrast, elevated concentrations of $\mathrm{Cu}$ were noted by Ghazaly et al. (1992) in the gills and trace amounts were accumulated in muscles of some commercial fishes from Alexandria. The relative accumulation of $\mathrm{Cu}$ in tissue of Oreochromis niloticus was of the order gills $>$ liver $>$ muscle (Birungi et al., 2007). Certain physiological processes may be stimulated by copper uptake, causing variable activity in various organs and tissue. This is particularly evident during blood production where copper is involved in haemoglobin synthesis (Avenant-Oldewage and Marx, 2000). For Zn, Shakweer and Abbas (1996) reported that the average concentrations of Zn were lower in the muscles of Oreochromis niloticus and Sardinella aurita than that in the livers, while Birungi et al. (2007) said that the relative accumulation of $\mathrm{Zn}$ in tissue of Oreochromis niloticus was of the order gills $>$ liver $>$ muscle. Working on $C$. gariepinus, $\mathrm{Zn}$ bioaccumulated in the different organs as: liver $>$ skin $>$ gills $>$ 
muscles (Gbem et al., 2001); liver > gills > kidney > muscles (Abdel-Baky, 2001); liver $>$ kidney $>$ gills $>$ skin $>$ muscles (Rizkalla et al, 2003) or gills $>$ liver $>$ kidney (Farombi et al., 2007). Also, Rizkalla et al. (2003) indicated that the main sites of storing Zn are kidney and spleen for Oreochromis species and spleen for Bagrus bayad and the lowest $\mathrm{Zn}$ concentration is recorded in the muscles of all fish species. For Mn, Abdel-Baky (2001) said that the concentration of Mn in different organs of C. gariepinus followed a sequence of gills $>$ liver $>$ kidney $>$ muscles. In general, AlNagaaway et al. (2009) mentioned that $\mathrm{Fe}, \mathrm{Zn}$ and $\mathrm{Cu}$ concentrations in Oreochromis niloticus organs were in the following order: liver $>$ gills $>$ muscles.

\section{CONCLUSION}

From the findings of this study it is concluded that the essential heavy metals of interest found in measurable quantities are still within safe limits for consumption. The metal concentrations in fish are the result of complex processes associated with uptake, excretion rates and homeostasis in fish. Essential heavy metals cannot easily be defined due to a number of factors that can influence and modify the toxicity of these substances. Some of these factors include: the metal species in the water; the presence of other metals; abiotic factors (i.e. temperature, $\mathrm{pH}$, dissolved oxygen, hardness, salinity, etc.); biotic factors (i.e. age, size and length of the fish, sex, stage in life history); feeding habits and behavioral responses. Through routine monitoring of the water at the sampling locations, the abiotic factors should be maintained at constant and acceptable levels and if this is not achieved, an increase in metal accumulation and toxicity would result which, ultimately, could be detrimental to the health of the fish. So, efforts should however be concentrated on ensuring that these concentrations are not exceeded. Safe disposal of domestic sewage and industrial effluents should be practiced and where possible recycled to avoid these metals and other contaminants from going into the environment. Laws enacted to protect our environment should be enforced.

\section{REFERENCES}

Abd El-Baky, T.E. (2001): Heavy metals concentrations in the catfish, Clarias gariepinus (Burchell, 1822) from River Nile, El-Salam Canal and Lake Manzala and their impacts on cortisol and thyroid hormones. Egypt. J. Aquati. Biol. \& Fish., 5 (1): 79-98.

Abd El-Khalek, A.M.; El-Shafi, M.A. and Abd El-Khalek, M. M. (2002): Monitoring of heavy metal residues in some freshwater fishes in Giza Governorate. Egypt. J. Agric. Res., 80 (2): 881-889.

Abd El-Nasser, M.; Shaaban, A.A.; Aly, S.M. and Sayed, M.M. (1996): Levels of some heavy metals in fish caught from river Nile at Assiut Governorate, Egypt. Assiut Vet. Med. J., 34 (68): 68-84.

Adeyeye, E.I.; Akinyugha, N .J.; Fesobi, M.E. and Tenabe, O. (1996): Determination of some metals in Clarias gariepinus (Cuvier and Vallenciennes), Cyprinus carpio (L) and Oreochromis niloticus (L) fishes in a polyculture fresh water pond and their environments. Aquacul., 147 (3-4): 205-214.

Adeyeye, E.I. and Otokiti, M.K.O. (1999): Proximate composition and some nutritionally valuable minerals of two varieties of Capsicum annum (bell and cherry pepper). Discovery and Innovation. 11 (1\&2): 75-81. 
Alabaster, J.S. and Lloyd, R. (1980): "Water Quality Criteria for Freshwater Fish". Butterworth and Co. Ltd., London, Great Britain. 297.

Al-Nagaaway, A.M.; Shehata,M.N.; Dawah, A.M. and Mansour, E.M. (2009): Evaluation of some heavy metals residues in different water sources and Nile Tilapia in Sharkia Governorate, Egypt. Egypt. J. Agric. Res., 87 (2): 591-600.

Al-Yousuf, M.H.; El-Shahawi, M.S. and Al-Ghais, S.M. (2000): Trace metals in liver, skin and muscle of Lethrinus lentjan fish species in relation to body length and sex. Sci. Total Environ., 256: 87-94.

Annune, P.A. and Iyaniwura, T.T. (1993): Accumulation of two trace metals in tissues of freshwater fishes, Oreochromis niloticus and Clarias gariepinus. J Aquat. Food Prod. Technol., 2 (3): 5-18.

Arain, M.B.; Kazi, T.G.; Jamali, M.K.; Jalbani, N.; Afridi, H.I. and Shah, A. (2008): Total dissolved and bioavailable elements in water and sediment samples and their accumulation in Oreochromis mossambicus of polluted Manchar Lake. Chemosphere, 70 (10): 1845-1856.

Ashraf, W. (2005): Accumulation of heavy metals in kidney and heart tissues of Epinephelus microdon fish from Arabian Gulf. Environ. Monit. Ass., 101: 311.

Avenant-Oldewage, A. and Marx, H.M. (2000): Bioaccumulation of chromium, copper and iron in the organs and tissues of Clarias gariepinus in the Olifants River, Kruger National Park. Water SA, 26 (4): 569-582.

Badsha, K.S. and Goldspink, C.R. (1982): Preliminary observations on the heavy metal content of four species of freshwater fishes in N.W. England. J Fish Biol., 21: 251-267.

Barlas, N. (1999): A pilot study of heavy metal concentration in various environments and fishes in the upper Sakarya River Basin, Turkey. Environ. Toxicol., 14 (3): 367-373.

Bebianno, M.J.; Geret, F.; Hoarau, P.; Serafim, M.A.; Coelho, M.R.; Gnassia-Barelli, M. and Romeo, M. (2004): Biomarkers in Ruditapes decussates: a potential bioindicator species. Biomarkers, 9: 305-330.

Beliles, A.A. (1979): The lesser metals. In "Toxicity of heavy metals in the Environment". (Ed. F.W. Oehme), Part II. Marcel Dekker Inc., N.Y., pp. 565597.

Birungi, Z.; Masola, B.; Zaranyika, M.F.; Naigaga, I. and Marshall, B. (2007): Active biomonitoring of trace heavy metals using fish (Oreochromis niloticus) as bioindicator species. The case of Nakivubo wetland along Lake Victoria. Phys. \& Chem. of the Earth, 32: 1350-1358.

Bohn, A. and McElroy, R.O. (1976): Trace metals in arctic cod and selected zooplankton from Strathcona Sound, Northern Baffin Island. J. Fish. Res. Bd. Can., 33: 2836.

Buckley, J.T.; Roch, M.; McCarter, J.A.; Rendell, C.A. and Matheson, A.T. (1982): Chronic exposure of coho salmon to sublethal concentrations of copper. I. Effect on growth, on accumulation and distribution of copper, and on copper tolerance. Comp. Biochem. Physiol., 72C (1): 15-19.

Carpene, E. and Vašák, M. (1989): Hepatic metallothionein from goldfish (Carassius auratus). Comp. Biochem. Physiol., 92B: 463-468.

Carpene, E.; Cattani, E.; Serrazanetti, P.; Fedrizzi, G. and Coretesi, P. (1990): Zinc and copper in fish from natural waters and rearing ponds in Northern Italy. J Fish Biol., 37: 293-299. 
Carvalho, C.S.; Araújo, H.S.S. and Fernandes, M.N. (2004a): Hepatic metallothionein in a teleost (Prochilodus scrofa) exposed to copper at $\mathrm{pH} 4.5$ and 8.0. Comp. Biochem. Physiol., 137B: 225-234

Carvalho, C.S.; Araújo, H.S.S. and Fernandes, M.N. (2004b): Copper toxicity and metallothionein induction in fish: effect of water temperature and $\mathrm{pH}$. Int. Cong. Biol. Fish, 215-223.

Chernoff, B. and Dooley,J.K. (1979): Heavy metals in relation to the biology of the mummichog, Fundulus heteroclitus. J. Fish Biol., 14 (3); 309-328.

Cid, B.P.; Boia, C.; Pombo, L. and Rebelo, E. (2001): Determination of trace metals in fish species of the Ria de Aveiro (Portugal) by electro thermal atomic absorption spectrometry. Food Chem., 75 (1): 93-100.

Clement Associates (1985): Chemical, physical and biological properties of compounds present at hazardous waste sites. Prepared for US Environmental Protection Agency, Washington, DC, pp. 312.

Coğun, H.Y. and Kargin, F. (2004): Effects of pH on the mortality and accumulation of copper in tissues of Oreochromis niloticus. Chemosphere, 55: 277-282.

Cotton, F. and Wilkinson, J. (1980): "Advanced Inorganic Chemistry". $4^{\text {th }}$ Ed., Wily Inter. Science: 724 pp.

Dallas, H.F. and Day, J.A. (1993): The effect of water quality variables on riverine ecosystems: A review. Fresh water Research Unit, University of Cape Town, Rondebosch, South Africa.

Daoud, J.R.; Amin, A.M. and Abd El-Khalek, M.M. (1999): Residual analysis of some heavy metals in water and Oreochromis niloticus fish from polluted areas. Vet. Med. J., Giza, 47 (3): 351-365.

DeOliveira, C.P.F.; Menezes, A.C.L. and Val, A.L. (2004): Effects of copper and lead on tambaqui, Colossoma macropomum (Cuvier, 1818). $\mathrm{VI}^{\text {th }}$ Symposium of "International Congress on the Biology of Fish". Manaus (Brazil) 1-5 August 2004, pp. 25-30.

Department of Water Affairs and Forestry (1993a): "South African Water Quality Guidelines. Vol. 1: Domestic Use". Department of Water Affairs and Forestry, Pretoria, South Africa. 216.

Department of Water Affairs and Forestry (1993b) "South African Water Quality Guidelines. Vol. 3: Industrial Use". Department of Water Affairs and Forestry, Pretoria, South Africa. 222.

Duffus, J.H. (1980): "Environmental Toxicology". Edward Arnold (publishers) Ltd., London, Great Britain. 164.

Du Preez, H.H. and Steyn, G.J. (1992): A preliminary investigation of the concentration of selected metals in the tissues and organs of the tigerfish (Hydrocynus vittatus) from the Olifants River, Kruger National Park, South Africa. Water SA, 18 (2): 131-136.

DWAF (1996): Procedures to assess effluent discharge impacts. Water Research Commission, South Africa. Report No. TT, pp. 64-351.

El-Nady, F. (1981): "Survey of Some Heavy Metals in Alexandria Water and its Effect on Some Marine Animals.” Ph.D. Thesis, Fac. of Sci., Alex. Univ.

El-Shehawi, A.M.; Ali, F.K. and Seehy, M.A. (2007): Estimation of water pollution by genetic biomarkers in tilapia and catfish species shows species-site interaction. African J. Biotechnol., 6 (7): 840-846.

Farombi, E.O.; Adelowo, O.A. and Ajimoko, Y.R. (2007): Biomarkers of oxidative stress and heavy metal levels as indicators of environmental pollution in African 
catfish (Clarias gariepinus) from Nigeria Ogun river. Int. J. Environ. Res. Public Health, 4 (2): 158-165.

Fast, A.W.; D’ltri, F.M.; Barclay, D.K.; Katase, S.A. and Madenjian, C. (1990): Heavy metal content of coho (Oncorhynchus kisutch) and Chinook salmon (O. tschawytscha) reared in deep upwelled ocean waters in Hawii. J. World Aqua. Soc., 21 (4): 271-276.

Förstner, U. and Wittmann, G.T.W. (1983): "Metal Pollution in the Aquatic Environment". Springer-Verlag, Berlin, Heidelberg, New york, Tokyo.

Friberg, L.; Nordberg, G.F. and Vouk, V.B. (1986): "Handbook on the Toxicology of Metals". Volume 11: Specific Metals. Elsevier Science Publishers, Amsterdam, Netherlands. 704.

Gaber, H.S. (2008): An investigation on health of Nile tilapia Oreochromis niloticus from Nile river ecosystem in Upper Egypt. Egypt. J. Aquat. Res., 34 (4): 331344.

Gardner, G.R. and LaRoche, G. (1973): Copper induced lesions in estuarian teleosts. J. Fish. Res. Bd. Can., 30: 363-368.

Gatlin, D.M. (III); Phillips, H.F. and Torrans, E.L. (1989): Effects of various levels of dietary copper and zinc on channel catfish. Aquacult., 76: 127-134.

Gatlin, D.M. (III); O’Connell, J.P. and Scarpa, J. (1991): Dietary zinc requirement of the red drum, Sciaenops ocellatus. Aquacult., 92: 259-265.

Gbem, T.T.; Balogun, J.K.; Lawal, F.A. and Annune, P.A. (2001): Trace metal accumulation in Clarias gariepinus (Teugels) exposed to sublethal levels of tannery effluent. Sci. Total Environ., 271 (1-3):1-9.

Ghazaly, K.S.; El-Mofty, M.M. and Attia, S.I. (1992): Effects of locality and tissue type on heavy metal levels of some commercial fishes from Alexandria, Egypt. Bull. Inst. Oceanogr. \& Fish. ARE, 18: 43-52.

Handy, R.D. (1996): Dietary exposure to toxic metals in fish. In: Taylor, E.W. (Ed.), "Toxicology of Aquatic Pollution". Cambridge University Press, Cambridge, pp. 29-60.

Harms, U. (1975): The levels of heavy metals (Mn, Fe, Co, Ni, Cu, Zn, Cd, Pb, Hg) in fish from onshore and offshore waters of the German bight. Z. Lebensm. Unters. Forschi., 157: 125-132.

Hassan, H.A. and Youssef, H. (1985): Determination of cadmium, copper and zinc in muscles of some freshwater fishes. Assiut Med. J., 9: 21-28.

Hassel, J.H.; Ney, J.J. and Garling, D.L. (1980): Heavy metals in a stream ecosystem at sites near highways. Trans. Am. Fish. Soc., 109 (6): 636-643.

Heath, A.G. (1987): "Water Pollution and Fish Physiology". CRC Press Inc., Boca Ranton, Florida, USA. P. 245.

Hem, I.D. (1989): "Study and Interpretation of the Chemical Characteristics of Natural Water". ( $3^{\text {rd }}$ edn.) United States Geological Survey Water-Supply Paper 2254. United States Government Printing Office, Washington DC, USA. 264.

Hogstrand, C. and Haux, C. (1991): Binding and detoxification of heavy metals in lower vertebrates with reference to metallothionein. Comp. Biochem. Physiol., 100C (1/2): 137-141.

Hogstrand, C.; Lithner, G. and Haux, C. (1991): The importance of metallothinein for the accumulation of copper, zinc and cadmium in environmentally exposed perch, Perca fluviatilis. Pharmacol. Toxicol., 68: 492-501.

Issa, Y.M.; Elewa, A.A.; Shehata, M.B. and Abd El-Satar, A.M. (1997): Factors affecting the distribution of some major and minor elements in River Nile at greater Cairo Area. Egypt. J. Anal. Chem., 6: 58-68. 
Jaffar, J. and Pervaiz, S. (1989): Investigation of multiorgan heavy metal trace metal content of meat of selected dairy, poultry, fowl and fish species in Pakistan. J. Sci. Ind. Res., 32: 175-177.

Janssens, T.K.S.; Roelofs, D. and van Straalen, N.M. (2009): Molecular mechanisms of heavy metal tolerance and evolution in invertebrates. Insect. Sci., 16: 3-18.

Jarup, L. (2003): Hazards of heavy metal contamination. Braz. Med. Bull., 68: 425462.

Jeng, S.S. and Sun, L.T. (1981): Effects of dietary levels on zinc concentrations in tissues of common carp. J. Neurol., 111: 134-140.

Jordao, C.P.; Pereira, M.G.; Bellato, C.R.; Pereira, J.L. and Matos, A.T. (2002): Assessment of water systems for contaminants from domestic and industrial sewages. En. viron Monit. Assess., 79 (1):75-100.

Karadede, H.; Oymakb, S.A. and Ünlü, E. (2004): Heavy metals in mullet, Liza abu, and catfish, Silurus triostegus, from the Atatürk Dam Lake (Euphrates), Turkey. Envir. Intern., 30: 183-188.

Kendrick, M.H.; Moy, M.T.; Plishka, M.J. and Robinson, K.D. (1992): "Metals and Biological Systems", Ellis Horwood Ltd., Chichester, UK.

Khalaf-Allah, S.S. (1998): Monitoring of heavy metal pollution in River Nile around Helwan, Egypt. J. Egypt. Vet. Med. Ass., 58 (4): 571-589.

Laurent, P. and Dunel, S. (1980): Morphology of gill epithelia in fish. Am. J. Physiol., 238: R147-R159.

Leatherland, J.F. and Woo, P.T.K. (1998): "Fish Diseases and Disorders. Vol. 2: NonInfectious Disorders". CABI Publishing, UK.

Liebman, H. (1960): Handbuch der Frischwasser-und Abwasser biologie, Bd. II, Munich, $1149 \mathrm{P}$.

Lloyd, R. (1992): "Pollution and Fresh Water Fish". Blackwell Scientific Publications Ltd., England.

Luckey, T.D. and Venugopal, B.T. (1977): A new classification system for toxic compounds. J. Toxicol. Environ. Health, 2: 633-638.

Mathis, B.J. and Kevern, N.R. (1975): Distribution of mercury, cadmium, lead and thallium in a eutrophic lake. Hydrobiologia, 46 (2/3): 207-222.

Mayer, G.D.; Leach, A.; Kling, P.; Olsson, P.E and Hogstrand, C. (2003): Activation of the rainbow trout metallothionein-A promoter by silver and zinc. Comp. Biochem. Physiol., 134B: 181-188.

Miller, P.A.; Lanno, R.P.; McMaster, M.E. and Dixon, D.G. (1993): Relative contributions of dietary and waterborne copper to tissue copper burdens and waterborne-copper tolerance in rainbow trout (Oncorhynchus mykiss). Can. J. Fish. Aquat. Sc., 50: 1683-1689.

Moore, J.W. and Ramamoorthy, S. (1984): "Heavy Metals in Natural Waters: Applied Monitoring and Impact Assessment". Springer-Verlag, New York, USA. 268.

Niazzy, Z.M.; Abd El-Aziz, T.; Abou El-Wafa, A.M.; Hussain, Z.A. and Amin, T.M. (1995): Evaluation of some heavy metals in serum and muscles in Clarias lazera in different locations in Egypt. Bull. High Inst. Public Health, 25 (2): 419-426.

Olaifa, F.E.; Olaifa, A.K.; and Onwude, T.E. $\left(2004_{\mathrm{a}}\right)$ : Lethal and sublethal effects of copper to the African catfish (Clarias gariepinus). African J. Biomed. Res., 7: 65-70.

Olaifa, F.E.; Olaifa, A.K.; Adelaja, A.A. and Owolabi, A.G. (2004b): Heavy metal contamination of Clarias gariepinus from a lake and fish farm in Ibadan, Nigeria. African J. Biomed. Res., 7: 145-148. 
Parker, C.R. (1972): "Water Analysis by Atomic Absorption Spectroscopy". Varian Techtron Ltd, Australia, No. 124.

Prosi, F. (1979): Heavy metals in aquatic organisms. In: Förstner, U. and Wittmann, G.T.W. (eds.) "Metal Pollution in the Aquatic Environment". Springer-Verlag, Berlin, Germany. 271-323.

Rao, L.M. and Padmaja, G. (2000): Bioaccumulation of heavy metals in $M$. cyprinoids from the harbor waters of Visakhapatnam. Bull. Pure Appl. Sci., 19 A (2): 77-85.

Rizkalla, E.H. and Abo Donia, M.A. (1996a): Some industrial heavy metal pollutants in River Nile and their effect on biological and non biological inhabitants. I. Essential heavy metals. Egypt. J. Agric. Res., 74 (1): 225-236.

Rizkalla, E.H. and Abo Donia, M.A. (1996b): Monitoring of some heavy metals in River Nile water in relation to their levels in tilapia in Upper Egypt. Egypt. J. Agric. Res., 74 (1): 239-250.

Rizkalla, E.H.; Haleem, H.H.; Sabri, A. and Hassan, A.A. (2003): A pilot and comparative study of heavy metals residues in tissues of fishes in relation with their levels in ambient water of Lake Mariut, a severely polluted lake on the Northwest coast of Egypt. J. Environ. Sci., 6 (4): 1065-1110. Special issue for the $7^{\text {th }}$ Nat. Conf. on Environmental Studies and Research "Towards a Better Environment: The Present and the Future", 20-22 April 2003.

Roméo, M.; Frasila, C.; Gnassia-Barelli, M.; Damien, G.; Micu, D. and Mustatal, G. (2005): Biomonitoring of trace metals in the Black Sea Romania. Water Res., 39: 596-604.

Saleh, M.A.; Saleh, M.A.; Fouda, M.M.; Saleh, M.A.; Abd El-Lattif, M.S. and Wilson, B.L. (1988): Inorganic pollution of the man-made lakes of Wadi ElRaiyan and its impact on aquaculture and wildlife of the surrounding Egyptian desert. Environ. Contam. Toxicol., 17: 391-403.

Seddek, A.S.H.; Salem, D.A.; El-Sawi, N.M. and Zaky, Z.M. (1996): Cadmium, Lead, nickel, copper, manganese and fluorine levels in River Nile Fish. Assiut. Vet. Med. J., 35 (69): 95-102.

Sellers, C.M.; Heath, A.G. and Bass, M.L. (1975): The effect of sublethal concentrations of copper and zinc on ventilatory activity. Blood oxygen and $\mathrm{pH}$ in rainbow trout (Salmo gairdneri). Water Res., 9: 401-408.

Seymore, T.; Du Preez, H.H. and Van Vuren, J.H.J. (1995): Manganese, lead, and strontium bioaccumulation in the tissues of the yellow fish Barbus marequensis from the lower Olifants river. Eastern Transvaai Water SA., 21 (2): 159-172.

Shakweer, L.M. and Abbas, M.M. (1996): Effect of sex on the concentration levels of some trace metals in Oreochromis niloticus of Lake Edko and Sardinella aurita of the Mediterranean waters, Egypt. Bull. Nat. Inst. Oceanogr. \& Fish., A.R.E., 22: 121-141.

Shakweer, L.M. and Abbas, M.M. (1997): Heavy metals concentration levels in some fish species of Lake Mariut and the Nozha Hydrodrome, Egypt during 1974 and 1995. Bull. Nat. Inst. Oceanogr. \& Fish., A.R.E., 23: 167-186.

Shaw, B.J. and Handy, R.D. (2006): Dietary copper exposure and recovery in Nile tilapia, Oreochromis niloticus. Aquatic Toxicology 76 (2): 111-121.

Snedecor, G.W. (1971): “Statistical Methods”. 14 th. (edn). Imes Iwa. The Iowa State Univ. Press., p: 593.

Stagg, R.M. and Shuttleworth, T.J. (1982): The accumulation of copper in Platichthys flesus L. and its effects on plasma electrolyte concentrations. J. Fish Biol., 20: 491-500. 
Stiff, M.J. (1971): The chemical states of copper in polluted fresh water and a scheme of analysis to differentiate them. Water Res., 5: 585-599.

USEPA (1991): "Test Method for Evaluating of Solid Waste". SW 846, US Environmental Protection Agency, Cincinnati. OH.

Vas, P.; Gordon, J.D.M.; Fielden, P.R. and Overnell, J. (1993): The trace metal ecology of ichthyofauna in the Rockall Trough, North-eastern Atlantic. Mar. Pollut. Bull., 26 (11): 607-612.

Viana, F.; Huertas, R. and Danulat, E. (2005): Heavy metal levels in fish from coastal waters of Uruguay. Arch. Environ. Contam. Toxicol., 48: 530-537.

Waalkees, N.C. (1998): Attachment A: summary of a workshop on metal based fuel additives. National Cancer Institute at NIEHS.

Walker, T.I. (1982): Effects of length and locality on the mercury content of blacklip abalone, blue mussel, sand flathead and long-nosed flathead from Port Phillip Bay, Victoria. Aust. J. Mar. Freswater Res., 33: 553-560.

Wepener, V.; Van-Vuren, J.H.J.; Du-Preez, H.H.(2001): Uptake and distribution of a copper, iron and zinc mixture in gill, liver and plasma of a freshwater teleost, Tilapia sparrmanii. Water, Soil \& Air, 27 (1): 99-108.

Wetzel, R.G. (1983): "Limnology" (2nd edn.) Saunders College Publishing, New York, USA. 767 pp.

WHO (1984): "WHO Bulletin Guide Line for Drinking Water, Water Quality". Vol.1.

Wood, C. and Van Vleet, E. (1996): Copper, cadmium and zinc in the liver, kidney and muscle tissues of bottlenose dolphins (Tursiops truncates) stranded in Florida. Mar. Pollut. Bull., 32 (12): 886-889.

\section{ARABIC SUMMARY}

$$
\begin{aligned}
& \text { تباين قيم المعادن الثقيلة فى أسماك القراميط المجمعة من شمال وجنوب منطقة القاهرة }
\end{aligned}
$$

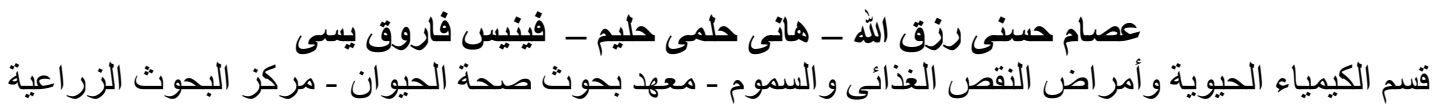

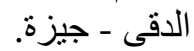

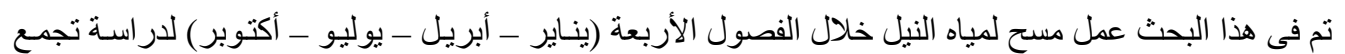

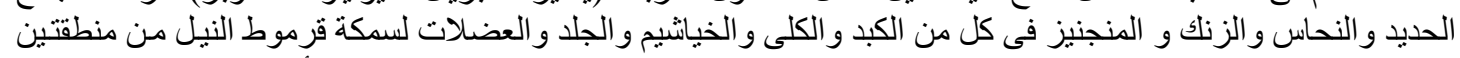

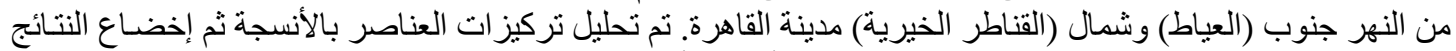

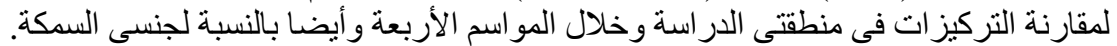

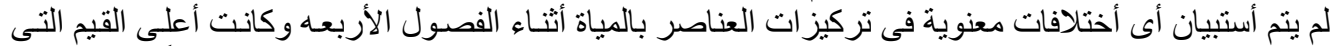

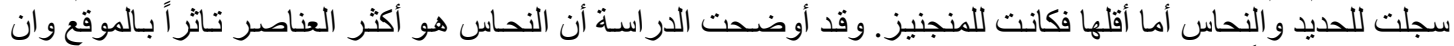

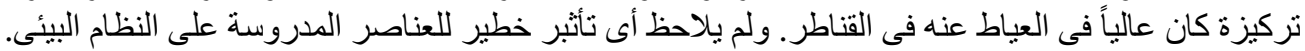

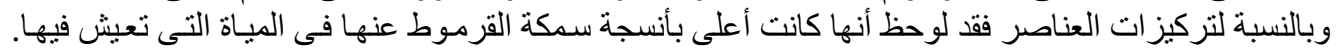

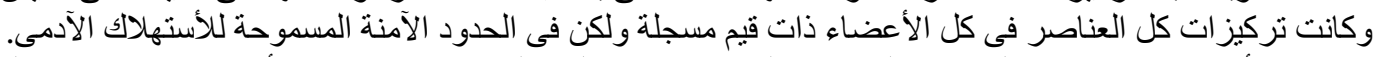

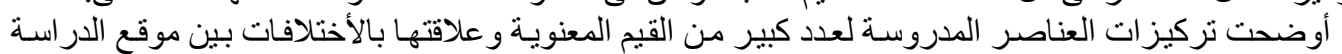

$$
\begin{aligned}
& \text { وجنس السكة و الموسم. وتم التركيز فى كتابة مناقثة البحث على هذة الاختلافات المعنوية. }
\end{aligned}
$$

